\title{
QUASI-REGULAR REPRESENTATIONS OF DISCRETE GROUPS AND ASSOCIATED $C^{*}$-ALGEBRAS
}

\author{
BACHIR BEKKA AND MEHRDAD KALANTAR
}

\begin{abstract}
Let $G$ be a countable group. We introduce several equivalence relations on the set $\operatorname{Sub}(G)$ of subgroups of $G$, defined by properties of the quasi-regular representations $\lambda_{G / H}$ associated to $H \in \operatorname{Sub}(G)$ and compare them to the relation of $G$ conjugacy of subgroups. We define a class $\operatorname{Sub}_{\mathrm{sg}}(G)$ of subgroups (these are subgroups with a certain spectral gap property) and show that they are rigid, in the sense that the equivalence class of $H \in \operatorname{Sub}_{\mathrm{sg}}(G)$ for any one of the above equivalence relations coincides with the $G$-conjugacy class of $H$. Next, we introduce a second class $\operatorname{Sub}_{\mathrm{w}-\text { par }}(G)$ of subgroups (these are subgroups which are weakly parabolic in some sense) and we establish results concerning the ideal structure of the $C^{*}$-algebra $C_{\lambda_{G / H}}^{*}(G)$ generated by $\lambda_{G / H}$ for subgroups $H$ which belong to either one of the classes $\operatorname{Sub}_{\mathrm{w}-\mathrm{par}}(G)$ and $\operatorname{Sub}_{\mathrm{sg}}(G)$. Our results are valid, more generally, for induced representations $\operatorname{Ind}_{H}^{G} \sigma$, where $\sigma$ is a representation of $H \in \operatorname{Sub}(G)$.
\end{abstract}

\section{INTRODUCTION}

Let $G$ be a countable discrete group. By results of Glimm [Gli61] and Thoma [Tho68], the classification of unitary dual $\widehat{G}$, that is, the set of irreducible unitary representations of $G$ up to unitary equivalence, is hopeless, unless $G$ is virtually abelian. By contrast, the primitive ideal space $\operatorname{Prim}(G)$, that is, the set of irreducible unitary representations of $G$ up to weak equivalence (see Section 7), is a more accessible dual space of $G$ : indeed, $\operatorname{Prim}(G)$, equipped with a natural Borel structure, is known to be a standard Borel space for any countable group $G$ (see [Eff63]).

Examples of irreducible unitary representations of $G$ are given by the quasi-regular representations $\left(\lambda_{G / H}, \ell^{2}(G / H)\right)$ of self-commensurating subgroups $H$ (see Subsection 2.6). Given two such subgroups $H$ and $L$, a natural question is: when do $\lambda_{G / H}$ and $\lambda_{G / L}$ define the same point in $\operatorname{Prim}(G)$, that is, when are $\lambda_{G / H}$ and $\lambda_{G / L}$ weakly equivalent? One

BB was supported by the Agence Nationale de la Recherche (ANR-11-LABX0020-01, ANR-14-CE25-0004); MK was supported by the NSF Grant DMS-1700259. 
of our main concerns in this paper is to study this question as well as related problems.

Let $\operatorname{Sub}(G)$ be the set of subgroups of $G$ and $\operatorname{Rep}(G)$ the set of unitary representations of $G$ on a separable Hilbert space. Consider the map

$$
\Lambda: \operatorname{Sub}(G) \rightarrow \operatorname{Rep}(G), \quad H \mapsto \lambda_{G / H}
$$

The group $G$ acts by conjugation on $\operatorname{Sub}(G)$ and $\Lambda$ factorizes to a map

$$
\operatorname{Sub}(G) / \sim_{\text {conj }} \rightarrow \operatorname{Rep}(G) / \sim_{\text {un }}
$$

where $\operatorname{Sub}(G) / \sim_{\text {conj }}$ is the set of conjugacy classes of subgroups of $G$ and $\operatorname{Rep}(G) / \sim_{\text {un }}$ is the set of equivalence classes of unitary representations of $G$.

The sets $\operatorname{Sub}(G)$ and $\operatorname{Rep}(G)$ carry natural topologies, respectively the Chabauty topology and the Fell topology. The map $\Lambda$ is continuous with respect to these topologies (see Proposition 3.3 below) and so factorizes to a map

$$
\operatorname{Sub}(G) / \sim_{\mathrm{w}-\text { conj }} \rightarrow \operatorname{Rep}(G) / \sim_{\mathrm{w}-\text { un }}
$$

where $\operatorname{Sub}(G) / \sim_{\mathrm{w}-\text { conj }}$ is the quasi-orbit space of $\operatorname{Sub}(G)$ with respect to the action of $G$ (see Section 3 ) and $\operatorname{Rep}(G) / \sim_{\text {w-un }}$ is the set of weak equivalence classes of unitary representations of $G$.

Unitary equivalence and weak equivalence of representations induce through the map $\Lambda: \operatorname{Sub}(G) \rightarrow \operatorname{Rep}(G)$ two other equivalence relations $\sim_{\text {rep }}$ and $\sim_{\text {w-rep }}$ on $\operatorname{Sub}(G):$

- $H \sim_{\text {rep }} L$ if $\lambda_{G / H}$ and $\lambda_{G / L}$ are equivalent;

- $H \sim_{\mathrm{w}-\text { rep }} L$ if $\lambda_{G / H}$ and $\lambda_{G / L}$ are weakly equivalent.

So, we have four equivalence relations $\sim_{\text {conj }}, \sim_{\mathrm{w}-\text { conj }}, \sim_{\text {rep }}$ and $\sim_{\mathrm{w}-\text { rep }}$ on $\operatorname{Sub}(G)$, the strongest and the weakest of which being $\sim_{\text {conj }}$ and $\sim_{\mathrm{w}-\mathrm{rep}}$, respectively. The following diagram summarizes the relationships between these equivalence relations (for more details, see Proposition 3.2 below):

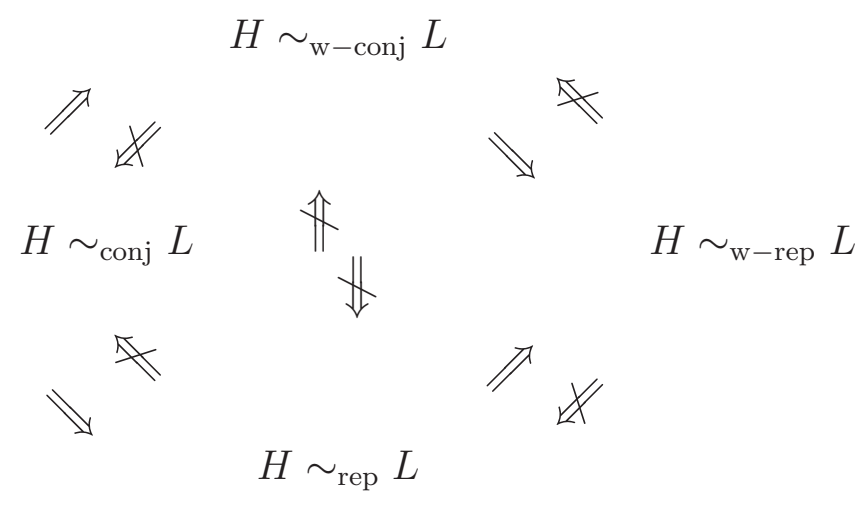


A natural question is: which subgroups $H$ of $G$ are rigid with respect to one of the relations $\sim_{\mathrm{w}-\text { conj }}, \sim_{\text {rep }}$, or $\sim_{\mathrm{w}-\text { rep }}$ in the sense that the equivalence class of $H$ with respect to one of these relations coincides with the conjugacy class of $H$ ? In particular, which subgroups have the strongest form of rigidity, that is, their $\sim_{\mathrm{w}-\mathrm{rep}}$-equivalence class and the conjugacy class coincide?

In this view, we introduce the class $\operatorname{Sub}_{\mathrm{sg}}(G)$ of what we call subgroups with the spectral gap property; this is the class of subgroups $H$ of $G$ such that the trivial representation $1_{H}$ is isolated in the spectrum of the natural representation of $H$ on $\ell^{2}(G / H)$. The class $\operatorname{Sub}_{\mathrm{sg}}(G)$ contains all subgroups with Kazhdan's property $(\mathrm{T})$ which are moreover strongly self-commensurating (see Definition 4.4 for this notion); the class $\operatorname{Sub}_{\mathrm{sg}}(G)$ contains also all non-amenable a-normal subgroups, that is, non-amenable subgroups $H$ of $G$ such that $H \cap g H^{-1}$ is amenable for every $g \in G \backslash H$. As an example, $H=S L_{n-1}(\mathbf{Z})$ belongs to $\operatorname{Sub}_{\mathrm{sg}}(G)$ for $G=S L_{n}(\mathbf{Z})$ and $n \geq 3$ (see Example 7.7); other examples include non-amenable maximal parabolic subgroups of convergence groups (see Proposition 5.15 below).

Our first result shows that subgroups from the class $\operatorname{Sub}_{\mathrm{sg}}(G)$ are rigid in the sense mentioned above.

Theorem A. Let $G$ be a countable group and $H \in \operatorname{Sub}_{\mathrm{sg}}(G)$. Then $H$ is representation rigid inside the class of self-commensurating subgroups of $G:$ if $L \in \operatorname{Sub}(G)$ is self-commensurating, then the representations $\lambda_{G / H}$ and $\lambda_{G / L}$ are weakly equivalent if and only if $L$ is conjugate to $H$.

Our next result deals with the ideal structure of the $C^{*}$-algebra $C_{\lambda_{G / H}}^{*}(G)$, that is, the closure in $\mathcal{B}\left(\ell^{2}(G / H)\right)$ of the linear span of $\lambda_{G / H}(G)$ for the norm topology (see Subsection 2.2). For subgroups $H \in \operatorname{Sub}_{\mathrm{sg}}(G)$, we prove the following result.

Theorem B. The $C^{*}$-algebra $C_{\lambda_{G / H}}^{*}(G)$ contains the ideal I of compact operators on $\ell^{2}(G / H)$ and $I$ is the smallest non-zero ideal of $C_{\lambda_{G / H}}^{*}(G)$ : every non-zero two-sided closed ideal of $C_{\lambda_{G / H}}^{*}(G)$ contains $I$.

Next, we introduce a second class of subgroups: the class $\operatorname{Sub}_{\mathrm{w}-\mathrm{par}}(G)$ of what we call weakly parabolic subgroups of $G$; this is the class of subgroups $H$ of $G$ for which there exists a topologically free boundary action $G \curvearrowright X$ such that $X$ admits an $H$-invariant probability measure (concerning the notion of a boundary action, see Subsection 5.2 below). When $G$ has a topologically free boundary action $G \curvearrowright X$, then every amenable subgroup are well as every point stabilizer of $X$ belongs to the class $\operatorname{Sub}_{\mathrm{w}-\text { par }}(G)$; see also Example 6.3. 
For the class of weakly parabolic subgroups, we prove the following result which generalizes a criterion from [KK17] for the reduced $C^{*}$ algebra $C_{\text {red }}^{*}(G)$ to be simple.

Theorem C. Let $G$ be a countable group and $H \in \operatorname{Sub}_{\mathrm{w}-\mathrm{par}}(G)$.

(i) The regular representation $\lambda_{G}$ factorizes to a representation of $C_{\lambda_{G / H}}^{*}(G)$; so, the reduced $C^{*}$-algebra $C_{\mathrm{red}}^{*}(G)$ of $G$ is isomorphic to $C_{\lambda_{G / H}}^{*}(G) / I$, where $I$ is the kernel of $\lambda_{G}$ in $C_{\lambda_{G / H}}^{*}(G)$.

(ii) $I$ is the largest proper ideal of $C_{\lambda_{G / H}^{*}}^{*}(G)$ : every proper two-sided closed ideal of $C_{\lambda_{G / H}^{*}}^{*}(G)$ is contained in $I$.

Theorem B has the following consequence for the space $\operatorname{Prim}(G)$.

Corollary D. Let $G$ be a countable group. The natural map

$$
\operatorname{Sub}_{\mathrm{sg}}(G) \rightarrow \operatorname{Prim}(G), \quad H \mapsto\left[\lambda_{G / H}\right]
$$

factorizes to an injective map from the quotient space $\operatorname{Sub}_{\mathrm{sg}}(G) / G$ to $\operatorname{Prim}(G)$, where $\left[\lambda_{G / H}\right]$ denotes the weak equivalence class of $\lambda_{G / H}$.

Actually, we prove much stronger versions of Theorem A, Theorem B, Theorem C, and Corollary D; indeed, our results are valid more generally for induced representations $\operatorname{Ind}_{H}^{G} \sigma$ for a representation $\sigma$ of $H$ : see respectively Theorem 4.3, Theorem 7.4, Theorem 7.2, and Theorem 7.1 below.

We apply our results to the case of subgroups arising as pointstabilizers of actions $G \curvearrowright X$ of $G$ on Hausdorff topological spaces $X$. In particular (see Theorem 5.1), we prove that, for the standard action $T \curvearrowright \mathbf{S}^{1}$ of Thompson's group $T$ on the circle and for $x, y \in \mathbf{S}^{1} \backslash\left\{e^{2 \pi i \theta} \mid \theta \in \mathbf{Q}\right\}$ which do not belong to the same $T$-orbit, the following hold:

$$
T_{x} \sim_{\mathrm{w}-\mathrm{conj}} T_{y} \quad \text { and } \quad T_{x} \nsim_{\text {rep }} T_{y},
$$

where $T_{x}$ and $T_{y}$ denote the stabilizers of $x$ and $y$ in $T$, respectively.

Interesting examples of subgroups $H$ which belong to either one of the classes $\operatorname{Sub}_{\mathrm{sg}}(G)$ or $\operatorname{Sub}_{\mathrm{w}-\mathrm{par}}(G)$ arise as point-stabilizers of actions $G \curvearrowright X$, where $X$ is extremally disconnected or where $G \curvearrowright X$ is a topologically free boundary action (see Section 5 below).

One example of a group $G$ and a subgroup $H$ which belong to both classes $\operatorname{Sub}_{\mathrm{sg}}(G)$ and $\operatorname{Sub}_{\mathrm{w}-\mathrm{par}}(G)$ is given by $G=P S L_{n}(\mathbf{Z})$ and $H=$ $P S L_{n-1}(\mathbf{Z})$ for $n \geq 3$ (see Example 7.7). Our results show in particular that $\operatorname{Prim}\left(P S L_{n}(\mathbf{Z})\right)$ is infinite for $n \geq 3$; this last result is also true for $n=2($ see $[\mathrm{BB} 18])$. 
This paper is organized as follows. Besides this introduction, the paper contains six more sections. In Section 2, we gather some background material on representation theory of discrete groups that will needed throughout the paper. We investigate in Section 3, connections between the equivalence relations introduced above and study rigidity properties of subgroups with respect to these equivalence relations. In Section 4, we prove Theorem 4.3 about rigidity of subgroups with the spectral gap property, which is a more general version of Theorem A. In Section 5, we study subgroups of a group $G$ which stabilize a point or a probability measure for a continuous action $G \curvearrowright X$. Section 6 is devoted to the study of weakly parabolic subgroups. In Section 7, we examine the ideal structure of $C^{*}$-algebras associated to induced representations; we prove there Theorem 7.4 and Theorem 7.2 which are more general versions of Theorem B and Theorem C.

\section{Preliminaries}

2.1. The compact space of subgroups. Let $G$ be a countable discrete group. Let $\operatorname{Sub}(G)$ be the set of subgroups of $G$, endowed with the Chabauty topology; this is the restriction to $\operatorname{Sub}(G)$ of the product topology on $\{0,1\}^{G}$, where every subgroup $H \in \operatorname{Sub}(G)$ is identified with its characteristic function $\mathbb{1}_{H} \in\{0,1\}^{G}$. The space $\operatorname{Sub}(G)$ is compact and metrizable, and the group $G$ acts continuously on $\operatorname{Sub}(G)$ by conjugation:

$$
G \times \operatorname{Sub}(G) \rightarrow \operatorname{Sub}(G), \quad(g, H) \mapsto g H g^{-1} .
$$

For $H \in \operatorname{Sub}(G)$, we denote by $\mathcal{C}(H)$ the $G$-orbit of $H$, that is, the conjugacy class of $H$. This gives rise to a first equivalence relation $\sim_{\text {conj }}$ on $\operatorname{Sub}(G)$ for which the equivalence class of $H \in \operatorname{Sub}(G)$ is $\mathcal{C}(H)$. We will introduce further equivalence relations on $\operatorname{Sub}(G)$ in Section 3.

2.2. $C^{*}$-algebras and von Neumann algebras associated to unitary representations. Let $G$ be a countable discrete group. Every unitary representation $(\pi, \mathcal{H})$ of $G$ extends uniquely to a representation (that is, a $*$-homomorphism) $\pi: \mathbf{C}[G] \rightarrow \mathcal{B}(\mathcal{H})$ of the group algebra $\mathrm{C}[G]$ of finitely supported functions on $G$ given by

$$
\pi(f)=\sum_{s \in G} f(s) \pi(s) \quad \text { for } \quad f \in \mathbf{C}[G] .
$$

The $C^{*}$-algebra $C_{\pi}^{*}(G)$ generated by $\pi$ is the norm closure in $\mathcal{B}(\mathcal{H})$ of the $*$-algebra $\{\pi(f) \mid f \in \mathbf{C}[G]\}$. The von Neumann algebra $W_{\pi}^{*}(G)$ generated by $\pi$ is the closure in $\mathcal{B}(\mathcal{H})$ of $C_{\pi}^{*}(G)$ for the strong (or weak) operator topology. 
Recall that the maximal $C^{*}$-algebra $C^{*}(G)$ of $G$ is the completion of $\mathbf{C}[G]$ of $G$ with respect to the norm

$$
f \mapsto \sup _{\pi \in \operatorname{Rep}(G)}\|\pi(f)\|
$$

where $\operatorname{Rep}(G)$ is the set of unitary representations of $G$ on a separable Hilbert space. Every $\pi \in \operatorname{Rep}(G)$ extends uniquely to a surjective *-homomorphism $\pi: C^{*}(G) \rightarrow C_{\pi}^{*}(G)$.

A unitary representation $(\pi, \mathcal{H})$ of $G$ is factorial if the von Neumann algebra $W_{\pi}^{*}(G)$ is a factor; in this case, $\pi$ is said to be traceable (or normal) if there exists a faithful normal (not necessarily finite) trace $\tau$ on $W_{\pi}^{*}(G)$ and a positive element $x \in C_{\pi}^{*}(G)$ such that $0<\tau(x)<+\infty$. If $\pi$ is irreducible, then $\pi$ is traceable if and only if the $C^{*}$-algebra $C_{\pi}^{*}(G)$ contains the algebra of compact operators on $\mathcal{H}$. For more details on all of this, see Chapters 6 and 17 in [Dix77].

For every subgroup $H$ of $G$, the injection map $i: H \rightarrow G$ extends to a $*$-homomorphism $i_{*}: C^{*}(H) \rightarrow C^{*}(G)$ which is injective, and so $C^{*}(H)$ can be viewed as a subalgebra of $C^{*}(G)$.

We denote by $\left(\lambda_{G}, \ell^{2}(G)\right)$ the (left) regular representation of $G$. The associated $C^{*}$-algebra $C_{\lambda_{G}}^{*}(G)$ is called the reduced $C^{*}$-algebra of $G$ and will be denoted by $C_{\text {red }}^{*}(G)$. The Dirac function $\delta_{e}$ at the neutral element $e \in G$ extends uniquely to a bounded trace $\tau_{G}$ on $W_{\lambda_{G}}^{*}(G)$, called the canonical trace on $G$.

2.3. Weak containment and Fell's topology. Let $G$ be a countable discrete group. Recall that $\rho \in \operatorname{Rep}(G)$ is weakly contained in $\pi \in$ $\operatorname{Rep}(G)$, in symbols $\rho \prec \pi$, if

$$
\|\rho(x)\| \leq\|\pi(x)\| \quad \text { for all } \quad x \in C^{*}(G),
$$

or, equivalently, if $C^{*} \operatorname{ker} \pi \subset C^{*} \operatorname{ker} \rho$, where $C^{*} \operatorname{ker} \pi$ and $C^{*} \operatorname{ker} \rho$ denote the kernels of the extensions of $\pi$ and $\rho$ to $C^{*}(G)$. The representations $\pi$ and $\rho$ are weakly equivalent, in symbols $\rho \sim \pi$, if $\pi \prec \rho$ and $\rho \prec \pi$.

We equip $\operatorname{Rep}(G)$ with Fell's topology (see Appendix F in [BdlHV08] or $[\operatorname{Dix} 77])$ : a sequence $\left(\pi_{n}\right)_{n}$ in $\operatorname{Rep}(G)$ converges to $\pi \in \operatorname{Rep}(G)$ if every function of positive type associated to $\pi$ is the pointwise limit of sums of functions of positive type associated to $\pi_{n}$ as $n \rightarrow \infty$; when $\pi$ is cyclic with cyclic vector $\xi$, it suffices to check this for the function of positive type $g \mapsto\langle\pi(g) \xi \mid \xi\rangle$ associated to $\xi$.

Convergence in $\operatorname{Rep}(G)$ can be expressed in terms of weak containment: $\lim _{n} \pi_{n}=\pi$ if and only if $\pi \prec \oplus_{k} \pi_{n_{k}}$ for every subsequence $\left(\pi_{n_{k}}\right)_{k}$ of $\left(\pi_{n}\right)_{n}$. 
2.4. States on $C^{*}$-algebras associated to unitary representations. We recall a few facts about states of $C^{*}$-algebras (for more details, see Chap. 2 in [Dix77]).

Let $\mathcal{A}$ be a unital $C^{*}$-algebra, with unit element $\mathbb{1}_{\mathcal{A}}$. A state of $\mathcal{A}$ is a positive linear functional $\varphi$ on $\mathcal{A}$ with $\varphi\left(\mathbb{1}_{\mathcal{A}}\right)=\|\varphi\|=1$. The set of states of $\mathcal{A}$, denoted $S(\mathcal{A})$, is a convex and compact subset of the unit ball of the dual space $\mathcal{A}^{*}$, where $\mathcal{A}^{*}$ is endowed with the weak* topology. Observe that in the case $\mathcal{A}=C(X)$ for a compact space $X$, the state space $S(\mathcal{A})$ is the space $\operatorname{Prob}(X)$ of probability measures on $X$.

Assume that $\mathcal{B}$ is a unital $C^{*}$ - subalgebra of $\mathcal{A}$. Then every state of $\mathcal{B}$ extends to a state of $\mathcal{A}$. If $\mathcal{C}$ is a quotient $C^{*}$-algebra of $\mathcal{A}$, then every state of $\mathcal{C}$ lifts to a state of $\mathcal{A}$ and so we can view $S(\mathcal{C})$ as weak* closed subset of $S(\mathcal{A})$.

Let $G$ be a discrete group and $\pi$ be a unitary representation of $G$. There is natural (right) action $G \curvearrowright S\left(C_{\pi}^{*}(G)\right)$ on the state space of $C_{\pi}^{*}(G)$ given by

$$
g \cdot \varphi(T)=\varphi\left(\pi(g) T \pi\left(g^{-1}\right)\right),
$$

for $g \in G, \varphi \in S\left(C_{\pi}^{*}(G)\right)$, and $T \in C_{\pi}^{*}(G)$.

Let $\pi, \rho$ be unitary representations of $G$. Viewing $S\left(C_{\pi}^{*}(G)\right)$ and $S\left(C_{\rho}^{*}(G)\right)$ as subsets of $S\left(C^{*}(G)\right)$, we have:

$$
\rho \prec \pi \Longleftrightarrow S\left(C_{\rho}^{*}(G)\right) \subset S\left(C_{\pi}^{*}(G)\right) .
$$

Assume, moreover, that $\rho$ is cyclic; then $\rho \prec \pi$ if and only if the state $T \mapsto\langle\rho(T) \xi \mid \xi\rangle$ belongs to $S\left(C_{\pi}^{*}(G)\right)$, where $\xi$ is a unit cyclic vector for $\rho$. In particular, for $\pi \in \operatorname{Rep}(G)$, we have

$$
\lambda_{G} \prec \pi \Longleftrightarrow \tau_{G} \in S\left(C_{\pi}^{*}(G)\right),
$$

where $\tau_{G} \in S\left(C_{\lambda}^{*}(G)\right.$ is the canonical trace on $G$.

2.5. A few facts about induced representations. Let $G$ be a countable discrete group. We collect a few known facts on induced representations $\operatorname{Ind}_{H}^{G} \sigma$ for $H \in \operatorname{Sub}(G)$ and for a unitary representation $(\sigma, \mathcal{K})$ of $H$. Recall that $\operatorname{Ind}_{H}^{G} \sigma$ of $G$ may be realized as follows. Let $\mathcal{H}$ be the Hilbert space of maps $F: G \rightarrow \mathcal{K}$ with the following properties

(i) $F(x h)=\sigma\left(h^{-1}\right) F(x)$ for all $x \in G, h \in H$;

(ii) $\sum_{x \in G / H}\|F(x)\|^{2}<\infty$.

The induced representation $\pi=\operatorname{Ind}_{H}^{G} \sigma$ is given on $\mathcal{H}$ by left translation:

$$
(\pi(g) F)(x)=F\left(g^{-1} x\right) \quad \text { for all } g \in G, F \in \mathcal{H} \text { and } x \in G .
$$


We give a decomposition of the restriction of $\operatorname{Ind}_{H}^{G} \sigma$ to another subgroup $L$ in terms of induced representations of $L$ and we recall how amenability of $H$ or $G$ can be expressed in terms of topological properties of $\operatorname{Ind}_{H}^{G} \sigma$.

For $H \in \operatorname{Sub}(G), \sigma \in \operatorname{Rep}(H)$ and $g \in G$, we denote by $\sigma^{g}$ the representation of $g^{-1} \mathrm{Hg}$ defined by

$$
\sigma^{g}(x)=\sigma\left(g x g^{-1}\right) \quad \text { for all } \quad x \in g^{-1} H g .
$$

The following lemma is well-known (for a proof, see for instance Proposition 9 in [BB18]).

Lemma 2.1. Let $G$ be a countable discrete group, $H, L \in \operatorname{Sub}(G)$ and $\sigma \in \operatorname{Rep}(H)$. Let $S$ be a system of representatives for the double coset space $H \backslash G / L$. The restriction of $\operatorname{Ind}_{H}^{G} \sigma$ to $L$ is equivalent to the direct sum

$$
\bigoplus_{s \in S} \operatorname{Ind}_{L \cap s^{-1} H s}^{L}\left(\left.\sigma^{s}\right|_{L \cap s^{-1} H s}\right) \text {. }
$$

Recall that, if $\pi$ is finite dimensional unitary representation of a group $G$, then $\pi \otimes \bar{\pi}$ contains the trivial representation $1_{G}$, where $\bar{\pi}$ is the conjugate representation of $\pi$, and $\pi \otimes \rho$ denotes the (inner) tensor product of the representations $\pi$ and $\rho$ (see [BdlHV08, Proposition A. $1.12])$.

Amenability of $G$ is characterized in terms of $\lambda_{G}$ by the HulanickiReiter theorem ([BdlHV08, Theorem G .3.2]):

$G$ is amenable $\Longleftrightarrow 1_{G} \prec \lambda_{G} \Longleftrightarrow \pi \prec \lambda_{G}$ for every $\pi \in \operatorname{Rep}(G)$.

Lemma 2.2. Let $\pi$ be a finite dimensional unitary representation of $G$. The following properties are equivalent:

(i) $\pi \prec \lambda_{G}$;

(ii) $G$ is amenable.

Proof. The fact that (ii) implies (i) follows from the Hulanicki-Reiter theorem. Assume that $\pi \prec \lambda_{G}$. Then

$$
\pi \otimes \bar{\pi} \prec \lambda_{G} \otimes \overline{\lambda_{G}} \sim \lambda_{G},
$$

where we used the fact that $\lambda_{G} \otimes \rho$ is equivalent to a multiple of $\lambda_{G}$ for every $\rho \in \operatorname{Rep}(G)$ (see e.g. [BdlHV08, Corollary E 1.12]). Since $\pi \otimes \bar{\pi}$ contains the trivial representation $1_{G}$, it follows that $1_{G} \prec \lambda_{G}$ and so $G$ is amenable, by the Hulanicki-Reiter theorem.

Here is a well-known consequence of Lemma 2.1 and Lemma 2.2.

Corollary 2.3. For $H \in \operatorname{Sub}(G)$ and $\sigma \in \operatorname{Rep}(H)$, the following properties are equivalent: 
(i) $\operatorname{Ind}_{H}^{G} \sigma \prec \lambda_{G}$;

(ii) $\sigma \prec \lambda_{H}$.

In particular, if $\sigma$ is finite dimensional, $\operatorname{Ind}_{H}^{G} \sigma \prec \lambda_{G}$ if and only if $H$ is amenable.

Proof. If $\sigma \prec \lambda_{H}$, then, by "continuity of induction" (see [BdlHV08, Theorem F.3.5]),

$$
\operatorname{Ind}_{H}^{G} \sigma \prec \operatorname{Ind}_{H}^{G} \lambda_{H}=\operatorname{Ind}_{H}^{G}\left(\operatorname{Ind}_{\{e\}}^{H} 1_{H}\right)=\lambda_{G} .
$$

Conversely, assume that $\operatorname{Ind}_{H}^{G} \sigma \prec \lambda_{G}$. Then $\left.\left.\left(\operatorname{Ind}_{H}^{G} \sigma\right)\right|_{H} \prec \lambda_{G}\right|_{H}$ and hence $\left.\left(\operatorname{Ind}_{H}^{G} \sigma\right)\right|_{H} \prec \lambda_{H}$, since $\left.\lambda_{G}\right|_{H}$ is a multiple of $\lambda_{H}$ (see [BdlHV08, Remark F.1.9]). By Lemma 2.1, $\sigma$ is a subrepresentation of $\left.\left(\operatorname{Ind}_{H}^{G} \sigma\right)\right|_{H}$. It follows that $\sigma \prec \lambda_{H}$.

Assume now that $\sigma$ is finite dimensional. Then, by Lemma 2.2, $\sigma \prec \lambda_{H}$ if and only if $H$ is amenable.

2.6. Irreducible quasi-regular representations. Let $G$ be a countable discrete group. Recall that $H, L \in \operatorname{Sub}(G)$ are commensurable if $H \cap L$ has finite index in both $H$ and $L$. The commensurator of $H$ in $G$ is the subgroup, denoted by $\operatorname{Comm}_{G}(H)$, of the elements $g \in G$ such that $g H^{-1}$ and $H$ are commensurable. The subgroup $H$ is self-commensurating if $\operatorname{Comm}_{G}(H)=H$.

For $H \in \operatorname{Sub}(G)$, denote by $\lambda_{G / H}$ the quasi-regular representation of $G$ on $\ell^{2}(G / H)$, that is, $\lambda_{G / H}=\operatorname{Ind}_{H}^{G} 1_{H}$.

The subgroups $H$ for which $\lambda_{G / H}$ is irreducible are described by the following classical result (see [Mac51], [Kle61], [Cor75]).

\section{Theorem 2.4. (Mackey's theorem)}

(i) Let $H$ be a self-commensurating subgroup of $G$ and let $\sigma$ a finite dimensional irreducible unitary representation of $H$. Then $\operatorname{Ind}_{H}^{G} \sigma$ is irreducible.

(ii) Let $H$ and $L$ be self-commensurating subgroups of $G$, and let $\sigma_{1}$ and $\sigma_{2}$ be finite dimensional irreducible unitary representations of $H$ and $L$, respectively. Then $\operatorname{Ind}_{H}^{G} \sigma_{1}$ and $\operatorname{Ind}_{L}^{G} \sigma_{2}$ are equivalent if and only if there exists $g \in G$ such that

- $g^{-1} L g=H$ and

- $\sigma_{1}$ is equivalent to $\sigma_{2}^{g}$.

Let $\widehat{G}$ be the unitary dual of $G$, that is, the set of equivalence classes of irreducible unitary representations of $G$.

Let $\operatorname{Sub}_{\mathrm{sc}}(G)$ be the subset of $G$ consisting of the self-commensurating subgroups of $G$. Set

$$
X:=\left\{(H, \sigma) \mid H \in \operatorname{Sub}_{\mathrm{sc}}(G), \sigma \in \widehat{H}_{\mathrm{fd}}\right\},
$$


where $\widehat{H}_{f d}$ denotes the set of equivalence classes of finite dimensional irreducible unitary representations of $H$. There is a natural (left) action of $G$ on $X$, given by

$$
(g,(H, \sigma)) \mapsto\left(g H g^{-1}, \sigma^{g^{-1}}\right) .
$$

Denote by $X / G$ the space of $G$-orbits in $X$. Identifying an irreducible representation of $G$ with its equivalence class, Theorem 2.4 can be rephrased as follows:

Theorem 2.5. The map

$$
\text { Ind }: X \rightarrow \widehat{G}, \quad(H, \sigma) \mapsto \operatorname{Ind}_{H}^{G} \sigma
$$

is well-defined and factorizes to an injective $\operatorname{map} X / G \rightarrow \widehat{G}$.

\section{RePRESEntation EQUivalent SUBGROUPS}

In this section, we introduce various equivalence relations on the space of subgroups of a discrete group, investigate connections between them, and study rigidity properties with respect to these equivalence relations.

3.1. Equivalence relations on the space of subgroups. Let $G$ be a countable discrete group and let $\operatorname{Sub}(G)$ be the set of subgroups of $G$, endowed with the Chabauty topology as in Subsection 2.1.

Definition 3.1. Let $H, L \in \operatorname{Sub}(G)$.

- $H$ and $L$ are weakly conjugate (in symbols, $H \sim_{\mathrm{w}-\text { conj }} L$ ) if $\overline{\mathcal{C}(H)}=\overline{\mathcal{C}(L)}$.

- $H$ and $L$ are representation equivalent (in symbols, $H \sim_{\text {rep }}$ $L)$ if the associated quasi-regular representations $\left(\lambda_{G / H}, \ell^{2}(G / H)\right)$ and $\left(\lambda_{G / L}, \ell^{2}(G / L)\right)$ are unitary equivalent.

- $H$ and $L$ are weakly representation equivalent (in symbols, $\left.H \sim_{\mathrm{w}-\text { rep }} L\right)$ if the associated quasi-regular representations $\left(\lambda_{G / H}, \ell^{2}(G / H)\right)$ and $\left(\lambda_{G / L}, \ell^{2}(G / L)\right)$ are weakly equivalent.

It is clear that all the relations introduced above are equivalence relations on $\operatorname{Sub}(G)$. We mention that all the equivalence relations we have introduced can be defined in exactly the same way on the space $\operatorname{Sub}(G)$ of closed subgroups of a general locally compact group $G$.

The relation of weak conjugacy is related to the notion of quasiorbits: given an action $G \curvearrowright X$ on a topological space $X$, consider the equivalence relation $\sim$ defined on $X$ by

$$
x \sim y \quad \text { if } \overline{G x}=\overline{G y} .
$$


An equivalence class for $\sim$ is called a quasi-orbit for $G$. The space $X / \sim$, equipped with the quotient topology, is a $\mathrm{T}_{0}$ topological space and is characterized as such by a certain universal property; quasiorbit spaces play an important rôle in the theory of $C^{*}$-algebras, among others. For more on this, see [Wil07, Chap.6].

The notion of representation equivalent subgroups (of general locally compact groups) appeared in the context of the question of the existence of isospectral but not isometric Riemannian manifolds (see [Sun85], [Gor09], [BPR14]).

We collect some facts about the equivalence relations on $\operatorname{Sub}(G)$ introduced in Definition 3.1.

Proposition 3.2. Let $H, L \in \operatorname{Sub}(G)$. Then

(i) $H \sim_{\text {conj }} L \Longrightarrow H \sim_{\mathrm{w}-\text { conj }} L$;

(ii) $H \sim_{\text {conj }} L \Longrightarrow H \sim_{\text {rep }} L$;

(iii) $H \sim_{\mathrm{w}-\text { conj }} L \Longrightarrow H \sim_{\mathrm{w}-\text { rep }} L$;

(iv) $H \sim_{\text {rep }} L \Longrightarrow H \sim_{\mathrm{w}-\text { rep }} L$;

(v) in general, $H \sim_{\mathrm{w}-\text { conj }} L \not \Longrightarrow H \sim_{\text {rep }} L$;

(vi) in general, $H \sim_{\text {rep }} L \not H \sim_{\text {conj }} L$;

(vii) in general, $H \sim_{\text {rep }} L \Rightarrow H \sim_{\text {w-conj }} L$;

(viii) in general, $H \sim_{\mathrm{w}-\mathrm{rep}} L \not H \sim_{\text {rep }} L$.

Proof. (i) and (iv) are obvious.

(ii) Assume that $H \sim_{\text {conj }} L$; we have to show that $\lambda_{G / H}$ is equivalent to $\lambda_{G / L}$. Indeed, if $L=g H^{-1}$ for $g \in G$, the map $U: \ell^{2}(G / H) \rightarrow$ $\ell^{2}(G / L)$, defined by

$$
U f(x)=f\left(g^{-1} x g\right) \quad \text { for } \quad f \in \ell^{2}(G / H), x \in G,
$$

is a bijective linear isometry. Moreover, $U$ intertwines $\lambda_{G / H}$ and $\lambda_{G / L}^{g^{-1}}$. As $\lambda_{G / L}^{g^{-1}}$ is unitary equivalent to $\lambda_{G / L}$, this proves the claim.

(iii) For the proof, see Corollary 3.4 below.

(v) For the proof, see Theorem 5.1 below.

(vi) There are examples of finite groups $G$ containing non conjugate subgroups $H, L$ such $\lambda_{G / H}$ and $\lambda_{G / L}$ are equivalent (see [Bér92, I.8]).

(vii) Let $G, H$ and $L$ be as in (vi); then $H \sim_{\text {rep }} L$ and $H \nsim$ conj $L$ and hence $H \chi_{\mathrm{w}-\operatorname{conj}} L$, since $G$ is finite.

(viii) Let $G=F_{2}$ be the free group on two generators $a, b$ and let $H$ be the subgroup generated by $a$ and $L=\{e\}$. On the one hand, $\lambda_{G / H}$ is weakly contained in $\lambda_{G}=\lambda_{G / L}$, since $H$ is amenable (see Corollary 2.3). On the other hand, $\lim _{n} b^{n} H b^{-n}=\{e\}$ and hence $\lambda_{G}$ is weakly contained in $\lambda_{G / H}$, by Corollary 3.4 below. Hence, we have 
$H \sim_{\mathrm{w}-\mathrm{rep}}\{e\}$. However, $\lambda_{G / H}$ is not equivalent to $\lambda_{G}=\lambda_{G / L}$. Indeed, $H$ has a non-zero invariant vector in $\ell^{2}(G / H)$ and no such vector in $\ell^{2}(G)$. Hence, $H \chi_{\text {rep }}\{e\}$.

3.2. Weak conjugacy and weak representation conjugacy. We investigate the relationship between weak conjugacy and weak representation conjugacy of subgroups of a countable discrete group $G$.

The following result is a special case of a far more general result of Fell, valid for induced representations of locally compact groups (see [Fel64, Theorem 4.2]).

Proposition 3.3. The map

$$
\Lambda: \operatorname{Sub}(G) \rightarrow \operatorname{Rep}(G), \quad H \mapsto \lambda_{G / H}
$$

is continuous.

Proof. Let $\left(H_{n}\right)_{n}$ be a sequence in $\operatorname{Sub}(G)$ converging to $H \in \operatorname{Sub}(G)$. Then $\mathbb{1}_{H_{n}}$ and $\mathbb{1}_{H}$ are functions of positive type on $G$ associated to $\lambda_{G / H_{n}}$ and $\lambda_{G / H}$, respectively; moreover, we have

$$
\lim _{n} \mathbb{1}_{H_{n}}(g)=\mathbb{1}_{H}(g) \quad \text { for every } \quad g \in G,
$$

by definition of the Chabauty topology on $\operatorname{Sub}(G)$. Since $\delta_{H} \in \ell^{2}(G / H)$ is a cyclic vector for $\lambda_{G / H}$ and since $\mathbb{1}_{H}=\left\langle\lambda_{G / H}(\cdot) \delta_{H} \mid \delta_{H}\right\rangle$, it follows that $\lim _{n} \lambda_{G / H_{n}}=\lambda_{G / H}$ in the Fell topology.

Corollary 3.4. Let $H, L \in \operatorname{Sub}(G)$.

(i) Assume that $L \in \overline{\mathcal{C}(H)}$. Then $\lambda_{G / L} \prec \lambda_{G / H}$.

(ii) $H \sim_{\mathrm{w}-\text { conj }} L \Longrightarrow H \sim_{\mathrm{w}-\text { rep }} L$.

Proof. (i) Let $L \in \overline{\mathcal{C}(H)}$, and let $H_{n} \in \mathcal{C}(H)$ be such that $\lim _{n} H_{n}=L$. Then, by continuity of the map $\Lambda$, it follows that

$$
\lim _{n} \lambda_{G / H_{n}}=\lim _{n} \Lambda\left(H_{n}\right)=\Lambda(L)=\lambda_{G / L} .
$$

Since $\lambda_{G / H^{\prime}}$ is equivalent to $\lambda_{G / H}$ for every $H^{\prime} \in \mathcal{C}(H)$, we have therefore $\lambda_{G / L} \prec \lambda_{G / H}$.

(ii) This follows immediately from Item (i) and the definitions.

Remark 3.5. (i) The converse in Corollary 3.4.i does not hold in general: for every amenable subgroup $H$ of $G$, we have $\lambda_{G / H} \prec$ $\lambda_{G}$ (see Corollary 2.3); however, $H \in \overline{\{e\}}$ only if $H=\{e\}$.

(ii) As shown in Proposition 3.2.vii, the converse in Corollary 3.4.ii does not hold in general. 
Let $\operatorname{Sub}_{\mathrm{a}}(G)$ be the subset of $\operatorname{Sub}(G)$ consisting of amenable subgroups of $G$. The following well-known fact (see [CM14, Corollary 4]) is another consequence of Proposition 3.3.

Corollary 3.6. Let $G$ be countable group. Then $\operatorname{Sub}_{\mathrm{a}}(G)$ is closed in $\operatorname{Sub}(G)$.

Proof. Let $\left(H_{n}\right)_{n}$ be a sequence in $\operatorname{Sub}_{\mathrm{a}}(G)$ converging to $H \in \operatorname{Sub}(G)$. Then $\lim _{n} \lambda_{G / H_{n}}=\lambda_{G / H}$, by Proposition 3.3. Since $H_{n}$ is amenable, we have $\lambda_{G / H_{n}} \prec \lambda_{G}$ for every $n$. Thus, we have $\lambda_{G / H} \prec \lambda_{G}$, which implies that $H$ is amenable (see Corollary 2.3).

3.3. Conjugation rigidity of subgroups. We define notions of rigidity for subgroups of a countable discrete group $G$, related to the various equivalence relations on $\operatorname{Sub}(G)$ introduced in Definition 3.1.

Definition 3.7. Let $H \in \operatorname{Sub}(G)$.

(i) $H$ is said to be conjugation rigid if, for every $L \in \operatorname{Sub}(G)$, we have

$$
L \sim_{\text {w-conj }} H \Longrightarrow L \sim_{\text {conj }} H
$$

(in other words, if $\overline{\mathcal{C}(L)}=\overline{\mathcal{C}(H)} \Longrightarrow \mathcal{C}(L)=\mathcal{C}(H)$ );

(ii) $H$ is said to be representation rigid if, for every $L \in \operatorname{Sub}(G)$, we have

$$
L \sim_{\text {w-rep }} H \Longrightarrow L \sim_{\text {conj }} H
$$

(in other words, if $\lambda_{G / L} \sim \lambda_{G / H} \Longrightarrow \mathcal{C}(L)=\mathcal{C}(H)$ );

(iii) $H$ is said to be strongly representation rigid if the following holds: if $\operatorname{Ind}_{L}^{G} \sigma \sim \operatorname{Ind}_{H}^{G} \pi$ for $L \in \operatorname{Sub}(G), \sigma \in \widehat{L}_{f d}$ and $\pi \in \widehat{H}_{f d}$, then there exists $g \in G$ such that $L=g^{-1} H g$ and $\sigma$ is equivalent to $\pi^{g}$.

Let $\mathcal{H}$ be a $G$-invariant subset of $\operatorname{Sub}(G)$ and $H \in \mathcal{H}$. One may define conjugation rigidity, representation rigidity and strong representation rigidity of $H$ inside $\mathcal{H}$, by allowing only subgroups $L \in \mathcal{H}$ in (i), (ii), and (iii).

Remark 3.8. Let $H \in \operatorname{Sub}(G)$.

(i) It follows from Corollary 3.4 that if $H$ is representation rigid, then $H$ is conjugation rigid.

(ii) $H$ may be conjugation rigid without being representation rigid; indeed, let $G=F_{2}$ be the free group on two generators $a, b$. The trivial subgroup $H=\{e\}$ is obviously conjugation rigid; however, for $L=\langle a\rangle$, the representation $\lambda_{G / L}$ is weakly equivalent to $\lambda_{G}$ (see proof of Proposition 3.2.viii), that is, $H \sim_{\mathrm{w}-\mathrm{rep}} L$. So, $H$ is not representation rigid. 
(iii) Generalizing (ii), let $G$ be $C^{*}$-simple group, that is, a group $G$ for which the reduced $C^{*}$-algebra $C_{\text {red }}^{*}(G)$ is simple; for large classes examples of such groups, see [BKKO17]). Then, for every amenable subgroup $L$ of $G$, we have $L \sim_{\text {w-rep }}\{e\}$; so, $H=\{e\}$ is conjugation rigid and not representation rigid if $G \neq\{e\}$.

\section{A Class OF RIGID SUbGROUPS}

We exhibit a class of subgroups $H$ of $G$ which have rigidity properties in the sense of Definition 3.7.

Let $G$ be a countable discrete group acting on a set $X$. Let $\pi_{X}$ be the associated natural representation of $G$ on $\ell^{2}(X)$. We say that the action $G \curvearrowright X$ has a spectral gap if $\pi_{X}$ does not weakly contain the trivial representation $1_{G}$; this is the case if and only if there exists no $G$-invariant mean on $\ell^{\infty}(X)$. For more on group actions with a spectral gap, see the overview [BB17].

Let $H \in \operatorname{Sub}(G)$. Observe that $H$ is a global fixed point for the natural action $H \curvearrowright G / H$.

Definition 4.1. We say that a subgroup $H \in \operatorname{Sub}(G)$ has the spectral gap property if the action $H \curvearrowright X$ has a spectral gap, where $X=$ $G / H \backslash\{H\}$. We denote by $\operatorname{Sub}_{\mathrm{sg}}(G)$ the set of all subgroups of $G$ with the spectral gap property.

Remark 4.2. (i) Let $H \in \operatorname{Sub}_{\mathrm{sg}}(G)$. Then $H$ is the only $H$ periodic point in $G / H$ (that is, the only point with finite $H$ orbit). Indeed, let $\mathcal{O} \subset G / H$ be a finite $H$-orbit. Then $\mathbb{1}_{\mathcal{O}}$ is an $H$-invariant non-zero function in $\ell^{2}(G / H)$ and hence $\mathcal{O}=\{H\}$.

(ii) By Lemma 2.1, a subgroup $H \in \operatorname{Sub}(G)$ has the spectral gap property if and only if $1_{H}$ is not weakly contained in the direct sum

$$
\bigoplus_{s \in G \backslash H} \lambda_{H /\left(H \cap s^{-1} H s\right)}
$$

(iii) Let $H \in \operatorname{Sub}_{\mathrm{sg}}(G)$. Then it follows from part (i) above that $H$ is self-commensurating. In fact, $H$ satisfies a slightly stronger property to be introduced in Definition 4.4 below.

We now establish a strong rigidity property of the class $\operatorname{Sub}_{\mathrm{sg}}(G)$. Recall that traceable representations have been defined in Subsection 2.2.

Theorem 4.3. Let $G$ be a countable discrete group and $H \in \operatorname{Sub}_{\mathrm{sg}}(G)$.

(i) Let $\sigma \in \widehat{H}_{f d}$. Then $\operatorname{Ind}_{H}^{G} \sigma$ is a traceable irreducible representation of $G$. 
(ii) Let $\sigma \in \widehat{H}_{f d}$ and $\pi \in \widehat{G}$. If $\pi \sim \operatorname{Ind}_{H}^{G} \sigma$, then $\pi$ is unitary equivalent to $\operatorname{Ind}_{H}^{G} \sigma$.

(iii) The subgroup $H$ is strongly representation rigid inside the class $\operatorname{Sub}_{\mathrm{sc}}(G)$ of self-commensurating subgroups: if $\operatorname{Ind}_{L}^{G} \sigma \sim \operatorname{Ind}_{H}^{G} \pi$ for $L \in \operatorname{Sub}_{\mathrm{sc}}(G), \sigma \in \widehat{L}_{f d}$ and $\pi \in \widehat{H}_{f d}$, then there exists $g \in G$ such that $H=g^{-1} \mathrm{Lg}$ and $\pi$ is equivalent to $\sigma^{g}$.

Proof. By Mackey's theorem (Theorem 2.4), Item (iii) is a special case of Item (ii). Item (ii) follows from Item (i), in combination with a wellknown fact about irreducible representations of $C^{*}$-algebras containing the algebra of compact operators (see Corollary 4.1.10 in [Dix77]). So, we only have to prove Item (i).

As a preliminary step, we first establish that $H$ enjoys a spectral gap property which is formally stronger than the one stated in Remark 4.2.ii.

- Step 1. For every $s \in G \backslash H$, let $\sigma_{s} \in \operatorname{Rep}\left(H \cap s^{-1} H s\right)$. We claim that the direct sum

$$
\rho:=\bigoplus_{s \in G \backslash H} \operatorname{Ind}_{H \cap s^{-1} H s}^{H} \sigma_{s}
$$

does not weakly contain any finite dimensional unitary representation of $H$.

Indeed, assume, by contradiction, that $\rho$ weakly contains a finite dimensional unitary representation of $H$. Then, $\rho \otimes \bar{\rho}$ weakly contains $1_{H}$. On the other hand, $\rho \otimes \bar{\rho}$ is equivalent to

$$
\bigoplus_{s \in G \backslash H} \operatorname{Ind}_{H \cap s^{-1} H s}^{H} \rho_{s}
$$

where $\rho_{s}=\sigma_{s} \otimes\left(\left.\bar{\rho}\right|_{H \cap s^{-1} H s}\right)$; see [BdlHV08, Proposition E. 2.5]. So, denoting by $\mathcal{K}_{s}$ the Hilbert space of $\rho_{s}$ for $s \in G \backslash H$, there exists a sequence $\left(F_{n}^{s}\right)_{n \geq 1}$ of maps $F_{n}^{s}: H \rightarrow \mathcal{K}_{s}$ in the Hilbert space of

$$
\pi_{s}:=\operatorname{Ind}_{H \cap s^{-1} H s}^{H} \rho_{s},
$$

with the following properties:

- $\sum_{s \in G \backslash H}\left\|F_{n}^{s}\right\|^{2}=1$ for all $n \geq 1$;

- $\lim _{n \rightarrow+\infty} \sum_{s \in G \backslash H}\left\|\pi_{s}(h) F_{n}^{s}-F_{n}^{s}\right\|^{2}=0$ for every $h \in H$.

Define, for every $s \in G \backslash H$, a sequence $\left(f_{n}^{s}\right)_{n \geq 1}$ of functions $f_{n}^{s}: H \rightarrow \mathbf{R}$ by

$$
f_{n}^{s}(x)=\left\|F_{n}^{s}(x)\right\| \quad \text { for } \quad x \in H
$$


Then $f_{n}^{s}$ is constant on the $H \cap s^{-1} H s$-cosets and, for the norm of $f_{n}^{s}$ in $\ell^{2}\left(H /\left(H \cap s^{-1} H s\right)\right)$, we have

$$
\left\|f_{n}^{s}\right\|^{2}=\sum_{x \in H /\left(H \cap s^{-1} H s\right)}\left\|F_{n}^{s}(x)\right\|^{2}=\left\|F_{n}^{s}\right\|^{2}
$$

hence,

$$
\sum_{s \in G \backslash H}\left\|f_{n}^{s}\right\|^{2}=\sum_{s \in G \backslash H}\left\|F_{n}^{s}\right\|^{2}=1
$$

for all $n \geq 1$. Moreover, for $s \in G \backslash H$ and $h \in H$, we have

$$
\begin{aligned}
\left\|\left(\lambda_{H /\left(H \cap s^{-1} H s\right)}\right)(h) f_{n}^{s}-f_{n}^{s}\right\|^{2} & =\sum_{x \in H /\left(H \cap s^{-1} H s\right)}\left\|F_{n}^{s}\left(h^{-1} x\right)\right\|-\left\|F_{n}^{s}(x)\right\| \|^{2} \\
& \leq \sum_{x \in H /\left(H \cap s^{-1} H s\right)}\left\|F_{n}^{s}\left(h^{-1} x\right)-F_{n}^{s}(x)\right\|^{2} \\
& =\left\|\pi_{s}(h) F_{n}^{s}-F_{n}^{s}\right\|^{2} .
\end{aligned}
$$

Therefore,

$$
\lim _{n \rightarrow+\infty} \sum_{s \in G \backslash H}\left\|\left(\lambda_{H /\left(H \cap s^{-1} H s\right)}\right)(h) f_{n}^{s}-f_{n}^{s}\right\|^{2}=0 .
$$

and so

$$
f_{n}:=\bigoplus_{s \in G \backslash H} f_{n}^{s}
$$

is a sequence of almost invariant unit vectors for the representation

$$
\bigoplus_{s \in G \backslash H} \lambda_{H /\left(H \cap s^{-1} H s\right)}
$$

this contradicts the fact that $H$ has the spectral gap property (see Remark 4.2.ii) and proves the claim.

- Step 2. Set $\pi:=\operatorname{Ind}_{H}^{G} \sigma$ for $\sigma \in \widehat{H}_{f d}$. We claim that $\pi$ is an irreducible and traceable representation of $G$.

Indeed, since $H$ is self-commensurating, the irreducibility of $\pi$ follows from Mackey's theorem. To prove that $\pi$ is traceable, it suffices to show that $\pi\left(C^{*}(G)\right)$ contains a non-zero compact operator.

Let $S \subset G$ be a system of representatives for the double coset space $H \backslash G / H$ with $e \in S$. By Lemma 2.1, $\left.\pi\right|_{H}$ is equivalent to

$$
\bigoplus_{s \in S} \operatorname{Ind}_{H \cap s^{-1} H s}^{H}\left(\left.\sigma^{s}\right|_{H \cap s^{-1} H s}\right)=\sigma \oplus \bigoplus_{s \in S \backslash\{e\}} \operatorname{Ind}_{H \cap s^{-1} H s}^{H}\left(\left.\sigma^{s}\right|_{H \cap s^{-1} H s}\right) .
$$


By Step 1 above, the representation

$$
\bigoplus_{s \in S \backslash\{e\}} \operatorname{Ind}_{H \cap s^{-1} H s}^{H}\left(\left.\sigma^{s}\right|_{H \cap s^{-1} H s}\right) .
$$

does not weakly contain the finite dimensional representation $\sigma$. It follows from [BB18, Lemma 6] that $\pi\left(C^{*}(H)\right)$ contains a non-zero compact operator. Since $C^{*}(H)$ can be viewed as subalgebra of $C^{*}(G)$, this proves the claim and hence Item (i).

4.1. Kazhdan subgroups. We now turn to examples of subgroups with the spectral gap property. We need the following strengthening of the notion of a self-commensurating subgroup.

Definition 4.4. A subgroup $H \in \operatorname{Sub}(G)$ is strongly self-commensurating in $G$ if $g H^{-1} \cap H$ has infinite index in $H$ for every $g \in G \backslash H$.

Remark 4.5. (i) It follows from Remark 4.2.i that every subgroup $H$ in $\operatorname{Sub}_{\mathrm{sg}}(G)$ is strongly self-commensurating in $G$.

(ii) A self-commensurating subgroup $H \in \operatorname{Sub}(G)$ is not necessarily strongly self-commensurating. Indeed, let $G=F_{2}$ be the free group on two generators $a$ and $b$. The subgroup $H$ generated by $\left\{b^{n} a b^{-n} \mid n \geq 1\right\}$ is easily seen to be self-commensurating. However, $b^{-1} H b \cap H=H$.

Our first main class of examples of subgroups with the spectral gap property is given by subgroups with Kazhdan's property (T); for an account on Kazhdan's property (T), see [BdlHV08].

Proposition 4.6. Let $H \in \operatorname{Sub}(G)$ be a strongly self-commensurating subgroup with property $(T)$. Then $H \in \operatorname{Sub}_{\mathrm{sg}}(G)$.

Proof. Assume, by contradiction, that the action $H \curvearrowright X$ does not have a spectral gap, where $X=G / H \backslash\{H\}$. Then (see Remark 4.2.ii) $1_{H}$ is weakly contained in the direct sum

$$
\bigoplus_{s \in G \backslash H} \lambda_{H / H \cap s^{-1} H s} .
$$

Since $H$ has property $(\mathrm{T})$, this implies $1_{H}$ is contained in $\lambda_{H / H \cap s^{-1} H s}$, for some $s \in G \backslash H$. Then $H \cap s^{-1} H s$ has finite index in $H$ and this contradicts the fact that $H$ is strongly self-commensurating.

It follows from Proposition 4.6 and Theorem 4.3 that strongly selfcommensurating Kazhdan subgroups are strongly representation rigid inside the class $\operatorname{Sub}_{s c}(G)$ of self-commensurating subgroups. We do not know whether such subgroups are representation rigid (or strongly representation rigid) inside the whole of $\operatorname{Sub}(G)$. However, as we now show, 
they are conjugation rigid. Indeed, as Kazhdan groups are finitely generated, this follows from the following proposition.

Proposition 4.7. Let $H$ be a strongly self-commensurating finitely generated subgroup of $G$. Then $H$ is conjugation rigid: if $L \in \operatorname{Sub}(G)$ is such that $\overline{\mathcal{C}(L)}=\overline{\mathcal{C}(H)}$, then $L$ is conjugate to $H$.

Proof. As $\overline{\mathcal{C}(L)}=\overline{\mathcal{C}(H)}$, we have $\lim _{n} x_{n} L x_{n}^{-1}=H$ and $\lim _{n} y_{n} H y_{n}^{-1}=$ $L$ for sequences $\left(x_{n}\right)_{n},\left(y_{n}\right)_{n}$ in $G$. Since $H$ is finitely generated, it follows from the definition of the Chabauty topology that there exists $n_{0} \geq 1$ such that $H \subset x_{n} L x_{n}^{-1}$ for every $n \geq n_{0}$. As

$$
\lim _{n} x_{n_{0}} y_{n} H y_{n}^{-1} x_{n_{0}}^{-1}=x_{n_{0}} L x_{n_{0}}^{-1}
$$

and, again, as $H$ is finitely generated, there exists $n_{1} \geq 1$ such that

$$
H \subset x_{n_{0}} y_{n} H y_{n}^{-1} x_{n_{0}}^{-1} \quad \text { for all } \quad n \geq n_{1} .
$$

Since $H$ is strongly self-commensurating, it follows that $x_{n_{0}} y_{n} \in H$, that is, $x_{n_{0}} y_{n} H y_{n}^{-1} x_{n_{0}}^{-1}=H$, for all $n \geq n_{1}$. Hence, $x_{n_{0}} L x_{n_{0}}^{-1}=H$.

Remark 4.8. The set of Kazhdan subgroups is in general neither closed nor open in $\operatorname{Sub}(G)$, as the following examples show.

- Let $G=\bigcup_{n \in \mathbf{N}} H_{n}$ be an inductive limit of a strictly increasing family of finite subgroups $H_{n}$. Then every $H_{n}$ has property $(\mathrm{T})$ but $\lim _{n} H_{n}=G$ does not have property (T), as it is not finitely generated.

- The sequence of the subgroups $H_{n}=2^{n} \mathbf{Z}$ of $G=\mathbf{Z}$, which of course do not have property $(\mathrm{T})$, converges to the Kazhdan subgroup $\{e\}$.

4.2. A-normal subgroups. We are going to introduce our second class of examples of subgroups with the spectral gap property.

Recall that a subgroup $H$ of $G$ is said to be malnormal (respectively weakly malnormal) if $H \cap g H^{-1}=\{e\}$ (respectively $H \cap g H g^{-1}$ is finite) for every $g \in G \backslash H$. For the relevance of this notion in group theory and operator algebras, see [LS01] and [PV08]). We introduce a class of subgroups which contains all weakly malnormal subgroups.

Definition 4.9. A subgroup $H \in \operatorname{Sub}(G)$ is said to be a-normal if $H \cap g \mathrm{Hg}^{-1}$ is amenable for every $g \in G \backslash H$. We denote by $\operatorname{Sub}_{\mathrm{a}-\text { norm }}(G)$ the set of all a-normal subgroups of $G$.

Remark 4.10. (i) Of course, every malnormal subgroup and every amenable subgroup is a-normal; however, we will be mainly interested in a-normal subgroups which are not amenable. 
(ii) Every non-amenable a-normal subgroup $H$ of $G$ is strongly selfcommensurating in $G$ in the sense of Definition 4.4: for $g \in$ $G \backslash H$, the group $H \cap g H g^{-1}$ is amenable and so cannot have finite index in the non-amenable group $H$.

Proposition 4.11. Let $H$ be a non-amenable a-normal subgroup of $G$. Then $H \in \operatorname{Sub}_{\mathrm{sg}}(G)$.

Proof. Assume by contradiction that $H$ is not in $\operatorname{Sub}_{\mathrm{sg}}(G)$, that is (see Remark 4.2), $1_{H}$ is weakly contained in the direct sum

$$
\bigoplus_{s \in G \backslash H} \lambda_{H / H \cap s^{-1} H s}
$$

Since $H$ is a-normal, $H \cap s^{-1} H s$ is amenable and so $\lambda_{H / H \cap s^{-1} H s}$ is weakly contained in $\lambda_{H}$ for every $s \in G \backslash H$ (see Corollary 2.3). This implies that $1_{H}$ is weakly contained in $\lambda_{H}$, hence that $H$ is amenable, and this is a contradiction.

It follows from Proposition 4.11 and Theorem 4.3 that non-amenable subgroups in $\operatorname{Sub}_{\mathrm{a}-\text { norm }}(G)$ are strongly representation rigid inside the class $\operatorname{Sub}_{s c}(G)$ of self-commensurating subgroups.

We do not know whether non-amenable a-normal subgroup are representation rigid (or strongly representation rigid) inside the whole of $\operatorname{Sub}(G)$. However, as we now show, they are conjugation rigid inside $\operatorname{Sub}(G)$. For this, we will need the following observation.

Lemma 4.12. The set $\operatorname{Sub}_{\mathrm{a}-\mathrm{norm}}(G)$ is a closed and $G$-invariant subset of $\operatorname{Sub}(G)$.

Proof. Since the conjugate of an a-normal subgroup is obviously again a-normal, $\operatorname{Sub}_{\mathrm{a}-\operatorname{norm}}(G)$ is $G$-invariant.

Let $\left(H_{n}\right)_{n}$ be a sequence in $\operatorname{Sub}_{\mathrm{a}-\text { norm }}(G)$ converging to $H \in \operatorname{Sub}(G)$. Let $x \in G \backslash H$. Since

$$
\lim _{n} H_{n} \cap x H_{n} x^{-1}=H \cap x H x^{-1}
$$

and since, by Corollary 3.6, $\operatorname{Sub}_{a}(G)$ is closed in $\operatorname{Sub}(G)$, it suffices to show that $x \notin H_{n}$ for $n$ large enough.

Assume, by contradiction, that $x \in H_{n_{k}}$ for a subsequence $\left(H_{n_{k}}\right)_{k}$ of $\left(H_{n}\right)_{n}$. Then $x \in H$, by the definition of the Chabauty topology, and this is a contradiction.

Corollary 4.13. Let $H$ be a non-amenable a-normal subgroup of $G$. Then $H$ is conjugation rigid: if $L \in \operatorname{Sub}(G)$ is such that $\overline{\mathcal{C}(L)}=\overline{\mathcal{C}(H)}$, then $L$ is conjugate to $H$. 
Proof. Let $L \in \operatorname{Sub}(G)$ be such that $\overline{\mathcal{C}(L)}=\overline{\mathcal{C}(H)}$. Then $L$ is a-normal, by Proposition 4.12. We claim that $L$ is non-amenable. Indeed, since $H \in \overline{\mathcal{C}(L)}$, it would follow otherwise that $H$ is amenable (see Corollary 3.6) and this contradicts our assumption. The claim now follows from Corollary 3.4 and Theorem 4.3.

\section{Point-Stabilizers of TOPOLOGICAL ACtions}

In this section, we study classes of subgroups of a group $G$ which arise as stabilizers of points for certain actions $G \curvearrowright X$ on a topological space $X$.

5.1. Continuity points of the stabilizer map. Given an action $G \curvearrowright X$ of $G$ on a Hausdorff topological space $X$ by homeomorphisms, let Stab : $X \rightarrow \operatorname{Sub}(G)$ be the $G$-equivariant map defined by

$$
\operatorname{Stab}(x)=G_{x} \quad \text { for } \quad x \in X,
$$

where $G_{x}=\{g \in G: g x=x\}$ is the stabilizer of $x$ in $G$.

For $x \in X$, denote by $G_{x}^{0}$ the (normal) subgroup of $G_{x}$ consisting of all $g \in G$ for which there exists a neighbourhood $U_{g}$ of $x$ such that $g y=y$ for all $y \in U_{g}$. It is easy to show that $\operatorname{Stab}: X \rightarrow \operatorname{Sub}(G)$ is continuous at $x \in X$ if and only if $G_{x}=G_{x}^{0}$; moreover, the set of continuity points of this map is a dense $G_{\delta}$-subset of $X$ if $X$ is a Baire space (for the elementary proof of these facts, see Lemmas 2.2 and Proposition 2.4 in [LBMB18]).

The following result, announced in Proposition 3.2, shows that, in particular, the equivalence relations $\sim_{\mathrm{w}-\text { conj }}$ and $\sim_{\text {rep }}$ on $\operatorname{Sub}(G)$ are not comparable in general.

Recall (see [CFP96]) that Thompson's group $T$ is the group of orientation preserving homeomorphisms of the circle $\mathbf{S}^{1}=\mathbf{R} / \mathbf{Z}$, which are piecewise linear, with only finitely many breakpoints, all at dyadic rationals, and with slopes all of the form $2^{k}$ for $k \in \mathbf{Z}$. Thompson's group $F$ is the stabilizer in $T$ of the point $1 \in \mathbf{S}^{1}$.

As is well-known and easy to check, the action $T \curvearrowright \mathbf{S}^{1}$ is minimal. Recall that an action $G \curvearrowright X$ of a group $G$ on a topological space $X$ is said to be minimal if the $G$-orbit $G x$ is dense in $X$ for every $x \in X$.

Theorem 5.1. Let $G$ be Thompson's group $T$ and consider its action on $\mathbf{S}^{1}$. Let $x, y \in \mathbf{S}^{1}$ and let $\sigma$ and $\rho$ be finite dimensional irreducible unitary representations of $G_{x}$ and $G_{y}$, respectively.

(i) The representation $\operatorname{Ind}_{G_{x}}^{G} \sigma$ is irreducible. 
(ii) The representations $\operatorname{Ind}_{G_{x}}^{G} \sigma$ and $\operatorname{Ind}_{G_{y}}^{G} \rho$ are equivalent if and only if there exists $g \in G$ such that $g x=y$ and $\rho$ is equivalent to $\sigma^{g^{-1}}$.

(iii) Assume that $y \notin G x$; then $G_{x} \chi_{\text {rep }} G_{y}$.

(iv) Assume that $x, y \in \mathbf{S}^{1} \backslash\left\{e^{2 \pi i \theta} \mid \theta \in \mathbf{Q}\right\}$; then $G_{x} \sim_{\mathrm{w}-\operatorname{conj}} G_{y}$.

Proof. Assume that $x \neq y$. We claim that $G_{x} \cap G_{y}$ has infinite index in $G_{x}$ or, equivalently, that the $G_{x}$-orbit of $y$ is infinite. Indeed, it follows from Lemma 4.2 in [CFP96] that every orbit of $G_{1} \cap G_{x}=F \cap G_{x}$ in $\mathbf{S}^{1} \backslash\{x, 1\}$ is infinite and it is clear that the $G_{x}$-orbit of 1 is infinite if $x \neq 1$.

Let $g \in G \backslash G_{x}$. Then, by what we have just shown, $G_{x} \cap g G_{x} g^{-1}=$ $G_{x} \cap G_{g x}$ has infinite index in $G_{x}$. Hence, $\operatorname{Comm}_{G}\left(G_{x}\right)=G_{x}$.

Moreover, we have $G_{y}=g G_{x} g^{-1}$ for some $g \in G$ if and only if $G_{y}=G_{g x}$, that is, if and only if $y=g x$. So, Items (i) and (ii) follow from Mackey's Theorem 2.4.

Item (iii) is a direct consequence of Item (ii).

To prove Item (iv), note that $\mathbf{S}^{1} \backslash\left\{e^{2 \pi i \theta} \mid \theta \in \mathbf{Q}\right\}$ is contained in the set $C$ of continuity points of the map $\operatorname{Stab}: \mathbf{S}^{1} \rightarrow \operatorname{Sub}(G)$.

Let $\mathcal{S}_{G}\left(\mathbf{S}^{1}\right)$ be the closure of $\left\{G_{z} \mid z \in C\right\}$ in $\operatorname{Sub}(G)$. Since $T \curvearrowright \mathbf{S}^{1}$ is minimal, the action $G \curvearrowright \mathcal{S}_{G}\left(\mathbf{S}^{1}\right)$ is minimal, by [Gla15, Proposition 1.2]. It follows that $G_{x} \sim_{\mathrm{w}-\text { conj }} G_{y}$ for all $x, y \in C$.

Remark 5.2. Consider the action of Thompson group $T$ on $\mathbf{S}^{1}$; the closure in $\operatorname{Sub}(T)$ of the set $\left\{T_{z} \mid z \in C\right\}$, which appeared in the proof of Theorem 5.1, is the stabilizer URS (short for "uniformly recurrent subgroup") for $T \curvearrowright \mathbf{S}^{1}$ in the sense of [Gla15]; this URS has been completely described in [LBMB18, Proposition 4.10].

We apply now the result (Proposition 3.3) on the continuity of the map

$$
\Lambda: \operatorname{Sub}(G) \rightarrow \operatorname{Rep}(G), \quad H \mapsto \lambda_{G / H}
$$

to point-stabilizers of a topological action of a group $G$.

Proposition 5.3. Let $G \curvearrowright X$ be an action of $G$ on a Hausdorff topological space $X$.

(i) Let $x \in X$ and let $z \in \overline{G x}$ be such that $G_{z}=G_{z}^{0}$. Then $G_{z}$ belongs to the closure of the conjugacy class $\mathcal{C}\left(G_{x}\right)$ of $G_{x}$; in particular, $\lambda_{G / G_{z}} \prec \lambda_{G / G_{x}}$.

(ii) Let $x \in X$ be such that $\overline{G x}$ contains a point with trivial stabilizer and $H$ be a subgroup of $G_{x}$. Then $\{e\}$ belongs to the closure of $\mathcal{C}(H)$ and $\lambda_{G} \prec \lambda_{G / H}$. 
Proof. For Item (i), note that by assumption, Stab : $X \rightarrow \operatorname{Sub}(G)$ is continuous at $z$ and $z \in \overline{G x}$. Thus, we have $G_{z} \in \overline{\mathcal{C}\left(G_{x}\right)}$, and Proposition 3.3 implies that $\lambda_{G / G_{z}} \prec \lambda_{G / G_{x}}$.

To show Item (ii), let $z \in \overline{G x}$ be such that $G_{z}=\{e\}$. We have trivially $G_{z}=G_{z}^{0}$; hence, $\{e\} \in \overline{\mathcal{C}\left(G_{x}\right)}$, by Item (i). It follows from the definition of the Chabauty topology that $\{e\} \in \overline{\mathcal{C}(H)}$ for every subgroup $H$ of $G_{x}$. This, together with Proposition 3.3, implies that $\lambda_{G} \prec \lambda_{G / H}$.

Recall that an action $G \curvearrowright X$ of $G$ on a Hausdorff topological space $X$ is called topologically free if, for every $g \in G \backslash\{e\}$, the set $\operatorname{Fix}(g)=$ $\{x \in X \mid g x=x\}$ of fixed points of $g$ has empty interior in $X$. Observe that, for such an action, the set of points $x \in X$ with $G_{x}=\{e\}$ is a dense (and in particular, non empty) subset of $X$, provided $X$ is a Baire space; indeed, the set of continuity points of the stabilizer map $X \rightarrow \operatorname{Sub}(G)$ is dense, as mentioned at the beginning of this section.

A Hausdorff topological space $X$ is called extremally disconnected (or Stonean) if the closure of every open set of $X$ is open. A relevant fact about these spaces is the following result from [Fro71].

Lemma 5.4. Let $X$ be an extremally disconnected topological space. The fixed point set of every homeomorphism of $X$ is open. In particular, if $G \curvearrowright X$ is an action of a group $G$ on $X$, then $G_{x}=G_{x}^{0}$ for every $x \in X$ and so the map Stab $: X \rightarrow \operatorname{Sub}(G)$ is continuous.

The following corollary is an immediate consequence of Lemma 5.4 and Proposition 5.3.

Corollary 5.5. Let $G \curvearrowright X$ be a minimal action of $G$ on an extremally disconnected topological space $X$. Then $G_{x} \sim_{\mathrm{w}-\mathrm{rep}} G_{y}$ for all $x, y \in X$.

5.2. Boundary actions. We turn our attention to subgroups which are point-stabilizers of boundary actions of a countable group $G$.

Let $X$ be a compact topological space. Recall that an action $G \curvearrowright X$ of $G$ on $X$ is said to be strongly proximal if, for every probability $\nu \in \operatorname{Prob}(X)$ on $X$, the weak* closure of the orbit $G \nu$ contains some point measure $\delta_{x}, x \in X$. The action $G \curvearrowright X$ is called a boundary action or a $G$-boundary if it is both minimal and strongly proximal.

By [Fur73], every group $G$ admits a universal boundary $\partial_{F} G$, called the Furstenberg boundary: $\partial_{F} G$ is a $G$-boundary and every $G$ boundary is a continuous $G$-equivariant image of $\partial_{F} G$. Moreover, it is known that $\partial_{F} G$ is extremally disconnected (see [KK17, Remark 3.16] or [BKKO17, Proposition 2.4]). 
It follows from Lemma 5.4 that, if $X$ is extremally disconnected, then $G \curvearrowright X$ is topologically free if and only if $G \curvearrowright X$ is free, that is, $\operatorname{Fix}(g)=\emptyset$ for every $g \in G \backslash\{e\}$.

$C^{*}$-simple groups have been characterized in [KK17] in terms their actions on boundaries as follows (see also [BKKO17]).

Theorem 5.6. [KK17] Let $G$ be a discrete group. The following properties are equivalent:

(i) $G$ is $C^{*}$-simple;

(ii) the action $G \curvearrowright \partial_{F} G$ is free;

(iii) there exists a topologically free boundary action $G \curvearrowright X$.

Recall that the amenable radical of a group $G$ is the unique largest amenable normal subgroup of $G$. A long well-known fact (see [BCdlH94, Lemma p.289]) is that a group with a non-trivial amenable radical is not $C^{*}$-simple; examples of non $C^{*}$-simple groups with a trivial amenable radical have been given in [LB17].

Proposition 5.7. Let $G$ be a countable group which is not $C^{*}$-simple and let $G \curvearrowright \partial_{F} G$ be the Furstenberg boundary action.

(i) For every $x \in \partial_{F} G$, the stabilizer $G_{x}$ is non-trivial and amenable.

(ii) For any $x, y \in \partial_{F} G$, we have $G_{x} \sim_{\mathrm{w}-\mathrm{conj}} G_{y}$.

(iii) For every $x \in \partial_{F} G$, we have $G_{x} \chi_{\mathrm{w}-\mathrm{rep}}\{e\}$.

(iv) Assume that the amenable radical of $G$ is trivial. Then there exists an uncountable subset $A$ of $\partial_{F} G$ such that, for every $x, y \in A$ with $x \neq y$, we have $G_{x} \chi_{\text {conj }} G_{y}$.

Proof. Since $G$ is not $C^{*}$-simple, Item (i) follows from Theorem 6.2 in [KK17] and from its proof (see also Theorem 3.1 and Proposition 2.7 in $[\mathrm{BKKO17]).}$

As $\partial_{F} G$ is extremally disconnected, the map $\operatorname{Stab}: \partial_{F} G \rightarrow \operatorname{Sub}(G)$ is continuous (see Lemma 5.4). Since $G \curvearrowright \partial_{F} G$ is minimal, the action of $G$ on $\left\{G_{x} \mid x \in \partial_{F} G\right\}$ is also minimal. This implies that $G_{x} \sim_{\mathrm{w}-\text { conj }} G_{y}$, for any pair of points $x, y \in \partial_{F} G$, and therefore proves Item (ii).

Let $x \in \partial_{F} G$. Since $G_{x}$ is non-trivial, it follows from [BKKO17, Proposition 3.5] that $\lambda_{G} \nprec \lambda_{G / G_{x}}$ and hence $G_{x} \chi_{\mathrm{w}-\mathrm{rep}}\{e\}$. This proves Item (iii).

By Item (i) and [LBMB18, Proposition 2.18(iii)], the set $\left\{G_{x} \mid x \in\right.$ $\left.\partial_{F} G\right\}$ is uncountable, provided $G$ has a trivial amenable radical. Since $G$ is countable, this implies Item (iv). 
5.3. A criterion for $C^{*}$-simplicity. We give a sufficient condition for the $C^{*}$-simplicity of a countable group $G$ in terms of a dynamical property of non necessarily compact $G$-spaces.

Proposition 5.8. Let $G \curvearrowright Y$ be a non-topologically free boundary action of $G$, and let $G \curvearrowright X$ be a topologically free minimal action of $G$ on a Baire space $X$. For every $y \in Y$, the subgroup $G_{y}$ has no global fixed point in $X$.

Proof. Since $G \curvearrowright Y$ is a non-topologically free boundary action, the quasi-regular representation $\lambda_{G / G_{y}}$ does not weakly contain the regular representation $\lambda_{G}$ for any $y \in Y$, by [BKKO17, Proposition 3.5].

Assume, by contradiction, that $G_{y}$ fixes a point $x \in X$. Since $G \curvearrowright$ $X$ is minimal and topologically free, $\overline{G x}=X$ contains a point with trivial stabilizer. Proposition 5.3 implies that $\lambda_{G} \prec \lambda_{G / G_{y}}$ and this is a contradiction.

Example 5.9. Let $T$ and $F$ be the Thompson groups.

(i) The standard action $T \curvearrowright \mathbf{S}^{1}$ is a non-topologically free boundary action and $F$ is a point-stabilizer for this action. Let $T \curvearrowright X$ be a topologically free minimal action of $T$ on a Baire space $X$. It follows from Proposition 5.8 that $F$ has no global fixed point in $X$.

(ii) Let $T \curvearrowright K$ be a minimal action of $T$ on a compact space $K$ such that $F$ stabilizes a point in $K$. The rigidity result on minimal actions of $T$ on compact spaces from [LBMB18, Theorem 1.8], combined with (i), shows that $T \curvearrowright K$ factors onto the standard action $T \curvearrowright \mathbf{S}^{1}$.

As a consequence of Proposition 5.8, we obtain a sufficient condition for $C^{*}$-simplicity of a group.

Corollary 5.10. Let $G$ be a group with the following property: for every amenable subgroup $H \in \operatorname{Sub}(G)$, there exists a topologically free minimal action $G \curvearrowright X$ on a Baire space $X$ such that $H$ fixes a point in $X$. Then $G$ is $C^{*}$-simple.

Proof. For each $y \in \partial_{F} G$, the stabilizer $G_{y}$ is amenable and hence fixes a point $x \in X$, by assumption. Proposition 5.8 implies that $G \curvearrowright \partial_{F} G$ is topologically free; therefore, $G$ is $C^{*}$-simple (see Theorem 5.6).

Example 5.11. Let $G$ be a torsion-free word hyperbolic group and let $G \curvearrowright \partial G$ be its the action on its Gromov boundary. Then every amenable subgroup of $G$ is infinite cyclic (see e.g. [KB02, Theorem $12.2])$ generated by a loxodromic element, hence fixes two points in $\partial G$. 
5.4. Examples of a-normal subgroups. In this subsection, we give some examples of classes of a-normal subgroups as defined in Subsection 4.2.

Example 5.12. Let $G=*_{i} H_{i}$ be a free product of countable discrete groups $H_{i}$. It follows from the definition of the free product that, for every factor group $H_{i}$ and every $s \notin H_{i}$, we have $s H_{i} s^{-1} \cap H_{i}=\{e\}$. Hence, every factor $H_{i}$ is an a-normal subgroup of $G$. If, in addition, a factor group $H_{i}$ is non-amenable, then $H_{i} \in \operatorname{Sub}_{\mathrm{sg}}(G)$.

We can determine when two factor groups in a free product are weakly representation equivalent.

Proposition 5.13. Let $G=H_{1} * H_{2}$ be the free product of non-trivial countable groups $\mathrm{H}_{1}$ and $\mathrm{H}_{2}$, at least one of which is of order bigger than two. Then $H_{1} \sim_{\mathrm{w}-\mathrm{rep}} H_{2}$ if and only if both $H_{1}$ and $H_{2}$ are amenable.

Proof. The group $G$ is $C^{*}$-simple (see for instance [PS79]). Assume that $H_{1}$ and $H_{2}$ are amenable. Then, by [BKKO17, Theorem 1.2], we have

$$
\lambda_{G / H_{1}} \sim \lambda_{G} \sim \lambda_{G / H_{2}} .
$$

Conversely, assume that $H_{1}$ is non-amenable. Since $H_{1}$ is a-normal (see Example 5.12), it follows $H_{1}$ is conjugation rigid, by Corollary 4.13. On the other hand, $H_{1} \chi_{\text {conj }} H_{2}$, by definition of the free product. Hence, $\lambda_{G / H_{1}} \chi_{\mathrm{w}-\mathrm{rep}} \lambda_{G / H_{2}}$.

The subgroups $H_{i}$ from Proposition 5.13 are examples of maximal parabolic subgroups of relatively hyperbolic groups, in the sense of Bowditch [Bow12], which in turn are special cases of convergence groups (see [Yam04] and [Bow98] for more details).

Definition 5.14. (i) A discrete group $G$ is a convergence group if it admits an action $G \curvearrowright X$ by homeomorphisms on a perfect compact Hausdorff space such that the induced action on the set of distinct triples $\{(x, y, z) \in X \times X \times X \mid \operatorname{Card}\{x, y, z\}=3\}$ is properly discontinuous. In this case, we say that $G \curvearrowright X$ is a convergence action.

(ii) Let $G$ be a convergence group. We say that a subgroup $H \in$ $\operatorname{Sub}(G)$ is parabolic if it is infinite and if there is a convergence action $G \curvearrowright X$ such that $H$ fixes some point of $X$ and such $H$ contains no loxodromic element, that is, no element $g \in G$ of infinite order with $\operatorname{Card}(\operatorname{Fix}(g))=2$.

If $G, H$, and $G \curvearrowright X$ are as in the above Definition 5.14.ii, then $H$ has a unique fixed point in $X$ which is called a parabolic point. 
The stabilizer of a parabolic point is a parabolic subgroup and hence a maximal parabolic subgroup.

Proposition 5.15. Let $G$ be a convergence group. Let $H \in \operatorname{Sub}(G)$ be a torsion-free maximal parabolic subgroup. Then $H$ is malnormal and hence a-normal. If, moreover, $H$ is non-amenable, then $H \in \operatorname{Sub}_{\mathrm{sg}}(G)$.

Proof. Since $H \in \operatorname{Sub}(G)$ is a maximal parabolic subgroup, there is a convergence action $G \curvearrowright X$ as in the Definition 5.14.ii, and a parabolic point $x \in X$ such that $H=G_{x}$. Let $g \in G \backslash H$, and let $s \in H \cap g H g^{-1}$. Then $s$ fixes the pair of distinct points $x$ and $g x$. Since $H$ does not contain any loxodromic elements, it follows $s$ has finite order and so $s=e$, as $H$ is torsion free. Thus, $H \cap g H g^{-1}=\{e\}$.

Here is another class of actions for which point-stabilizers are anormal (and not necessarily malnormal).

Proposition 5.16. Let $G \curvearrowright X$ be an action of the group $G$ on a set $X$. Assume that no non-amenable subgroup of $G$ fixes more than one point in $X$. Then $G_{x}$ is a-normal for every $x \in X$.

Proof. Let $x \in X$ and $g \in G \backslash G_{x}$. Then $g x \neq x$; the subgroup $G_{x} \cap g G_{x} g^{-1}$, which fixes both $x$ and $g x$, is therefore amenable.

Example 5.17. Let $\mathbf{K}$ be a field and $G$ a subgroup of $G L_{3}(\mathbf{K})$. The standard action $G \curvearrowright X$ on the projective plane $X=\mathbf{P}^{2}(\mathbf{K})$ satisfies the condition of Proposition 5.16 above; indeed, for distinct points $x$ and $y$ in $X$, the group $G_{x} \cap G_{y}$ is conjugate inside $G L_{3}(\mathbf{K})$ to a subgroup of the group

$$
L:=\left[\begin{array}{lll}
* & 0 & * \\
0 & * & * \\
0 & 0 & *
\end{array}\right]
$$

since $L$ is amenable, it follows that $G_{x} \cap G_{y}$ is amenable. Hence $G_{x}$ is a-normal for every point $x \in X$.

\section{WEAKLY PARABOLIC SUBGROUPS}

In this section, we study subgroups of a group $G$ which stabilize a probability measure on a $G$-boundary.

\subsection{Weakly parabolic subgroups: definition and examples.} Let $X$ be a compact space. Recall that the space $\operatorname{Prob}(X)$ of probability measures on $X$, endowed with the weak* topology, is a compact space. Assume that we have an action $G \curvearrowright X$ of a group $G$ on $X$. Then $G$ acts on $\operatorname{Prob}(X)$ through $\nu \mapsto g_{*} \nu$, where $g_{*} \nu$ is the image of $\nu \in \operatorname{Prob}(X)$ under the map $x \mapsto g x$ for $g \in G$.

We introduce our second main class of subgroups of $G$. 
Definition 6.1. Let $G$ be a countable group. We say that a subgroup $H$ of $G$ is weakly parabolic, if there exists a topologically free boundary action $G \curvearrowright X$ such that $H$ has a fixed point in $\operatorname{Prob}(X)$. We denote by $\operatorname{Sub}_{\mathrm{w}-\text { par }}(G)$ the set of weakly parabolic $H \in \operatorname{Sub}(G)$.

Remark 6.2. Let $G$ be a group which admits a topologically free boundary action $G \curvearrowright X$.

(i) The group $G$ is $C^{*}$-simple, by Theorem 5.6.

(ii) The set

$$
\{H \in \operatorname{Sub}(G) \mid H \text { has a fixed point in } \operatorname{Prob}(X)\}
$$

is a closed and $G$-invariant subset of $\operatorname{Sub}(G)$. It follows that $\operatorname{Sub}_{\mathrm{w}-\mathrm{par}}(G)$ is a $G$-invariant subset of $\operatorname{Sub}(G)$.

Example 6.3. (i) Let $G$ be a group which admits a topologically free boundary action. Then every amenable subgroup of $G$ belongs to $\operatorname{Sub}_{\mathrm{w}-\text { par }}(G)$. Indeed, if $H \curvearrowright X$ is an action of an amenable group on a compact space $X$, then $H$ has a fixed point in $\operatorname{Prob}(X)$.

(ii) Let $G \curvearrowright X$ be a topologically free boundary action of a group $G$. Let $x \in X$. Every subgroup of the point-stabilizer $G_{x}$ of $x$ is weakly parabolic. Here is a specific example.

- Let $G=P G L_{n}(\mathbf{Q})$ for $n \geq 2$ and let $G \curvearrowright X$ be the standard action on the projective space $X=\mathbf{P}\left(\mathbf{k}^{n}\right)$, where $\mathbf{k}$ is the field $\mathbf{R}$ of real numbers or the field $\mathbf{Q}_{p}$ of $p$-adic numbers. Let $x$ be the image in $\mathbf{P}\left(\mathbf{k}^{n}\right)$ of some vector from $\mathbf{Q}^{n} \backslash\{0\}$. Then the point stabilizer $G_{x}$ is isomorphic to the semi-direct product $P G L_{n-1}(\mathbf{Q}) \ltimes \mathbf{Q}^{n-1}$, given by the standard linear action on $\mathrm{Q}^{n-1}$.

(iii) Let $\mathbf{G} \curvearrowright X$ be a continuous action of a locally compact group $\mathbf{G}$ on a compact space $X$. Let $G$ be a countable (not necessarily discrete) subgroup of $\mathbf{G}$ such that the restricted action $G \curvearrowright X$ is a topologically free boundary action of $G$. Let $\mathbf{H}$ be an amenable closed subgroup of G. Then every subgroup $H$ of $G \cap \mathbf{H}$ belongs to $\operatorname{Sub}_{\mathrm{w}-\mathrm{par}}(G)$. Indeed, the locally compact amenable group $\mathbf{H}$ stabilizes a probability measure on $X$ and the claim follows. Here are two specific examples.

- For $n \geq 2$, we claim that $H=P O_{n}(\mathbf{Q})$, the (projective) group of orthogonal matrices with rational entries, is a weakly parabolic subgroup of $G=P G L_{n}(\mathbf{Q})$. Indeed, let $\mathbf{G}=P G L_{n}(\mathbf{R})$ and $\mathbf{G} \curvearrowright X$ the standard action on $X=\mathbf{P}\left(\mathbf{R}^{n}\right)$. The action $G \curvearrowright X$ is a topologically free boundary action and $H$ is contained in the compact subgroup $P O_{n}(\mathbf{R})$ of $\mathbf{G}$. Observe that $H$ is not amenable when $n \geq 3$, as it is dense in $P O_{n}(\mathbf{R})$ and so contains non abelian free subgroups. 
- For $n \geq 2$, we claim that $H=P S L_{n}(\mathbf{Z})$ is a weakly parabolic subgroup of $G=P S L_{n}(\mathbf{Q})$. Indeed, let $\mathbf{G}=P S L_{n}\left(\mathbf{Q}_{p}\right)$ for a prime integer $p$ and $\mathbf{G} \curvearrowright X$ the standard action on $X=\mathbf{P}\left(\mathbf{Q}_{p}^{n}\right)$. The action $G \curvearrowright X$ is a topologically free boundary action and $H$ is contained in the compact subgroup $P S L_{n}\left(\mathbf{Z}_{p}\right)$ of $\mathbf{G}$.

6.2. Some properties of weakly parabolic subgroups. The following property of weakly parabolic subgroups will be a crucial tool in the study of their associated $C^{*}$-algebras (see the proof of Theorem 7.2). Recall that a map $\Phi: \mathcal{A} \rightarrow \mathcal{B}$ between two unital $C^{*}$-algebras is unital if $\Phi\left(\mathbb{1}_{\mathcal{A}}\right)=\mathbb{1}_{\mathcal{B}}$ and that $\Phi$ is positive if $\Phi(T) \geq 0$ for every $T \in \mathcal{A}$ with $T \geq 0$.

Proposition 6.4. Let $G$ be a countable group and $H \in \operatorname{Sub}_{\mathrm{w}-\mathrm{par}}(G)$. Then there exist a topologically free boundary action $G \curvearrowright X$ and a linear isometric map $C(X) \rightarrow \ell^{\infty}(G / H)$ which is $G$-equivariant, unital and positive.

Proof. Since $H \in \mathrm{Sub}_{\mathrm{w}-\mathrm{par}}(G)$, there exists a topologically free boundary action $G \curvearrowright X$ such that $X$ admits an $H$-invariant probability measure $\nu$. Consider the Poisson transform $\mathcal{P}_{\nu}: C(X) \rightarrow \ell^{\infty}(G / H)$, defined by

$$
\mathcal{P}_{\nu}(f)(g H)=\int_{X} f(g x) d \nu(x) \quad \text { for } \quad f \in C(X), g \in G .
$$

It is clear that $\mathcal{P}_{\nu}$ is linear, $G$-equivariant, unital and positive. So, $\mathcal{P}_{\nu}$ induces a $G$-equivariant map $\Phi: G / H \rightarrow \operatorname{Prob}(X)$, given by

$$
\Phi(g H)(f)=\mathcal{P}_{\nu}(f)(g H) \quad \text { for } \quad f \in C(X), g \in G .
$$

Since $G \curvearrowright X$ is a boundary action, the range of $\Phi$ consists exactly of the point measures $\delta_{x}$ for $x \in X$ (see [Fur73, Proposition 4.2]). So, there exists a surjective map $\varphi: G / H \rightarrow X$ such that $\Phi(g H)=\delta_{\varphi(g H)}$ for every $g \in G$. It follows that $\mathcal{P}_{\nu}$ is isometric; indeed, let $f \in C(X)$. Then

$$
\begin{aligned}
\sup _{g \in G}\left|\mathcal{P}_{\nu}(f)(g H)\right| & =\sup _{g \in G}|\Phi(g H)(f)| \\
& =\sup _{g \in G}|f(\varphi(g H))| \\
& =\sup _{x \in X}|f(x)| .
\end{aligned}
$$

The next result generalizes the well-known fact that a $C^{*}$-simple group contains no non-trivial amenable normal subgroup. Recall that, if $\operatorname{Sub}_{\mathrm{w}-\mathrm{par}}(G) \neq \emptyset$, then $G$ is $C^{*}$-simple (Remark 6.2). 
Proposition 6.5. Let $G$ be a countable group. Then $\operatorname{Sub}_{\mathrm{w}-\mathrm{par}}(G)$ contains no non-trivial normal subgroup of $G$.

Proof. Let $N$ be a normal subgroup of $G$, and suppose there is a topologically free boundary action $G \curvearrowright X$ and an $N$-invariant $\nu \in$ $\operatorname{Prob}(X)$. Since $N$ is normal, $g_{*} \nu$ is $N$-invariant for every $g \in G$. As $G \curvearrowright X$ is a boundary action, the point measure $\delta_{x}$ belongs to the the weak*-closure of $\left\{g_{*} \nu \mid g \in G\right\}$ for every $x \in X$. Hence, $N$ acts trivially on $X$ and this implies that $N=\{e\}$, since $G \curvearrowright X$ is topologically free.

Next, we establish an interesting property of the conjugacy class of weakly parabolic subgroups.

Theorem 6.6. Let $G$ be a countable group. For $H \in \operatorname{Sub}_{\mathrm{w}-\mathrm{par}}(G)$, the following hold:

(i) $\{e\} \in \overline{\mathcal{C}(H)}$;

(ii) $\lambda_{G} \prec \lambda_{G / H}$.

Proof. Let $G \curvearrowright X$ be a topologically free boundary action such that $H$ stabilizes a probability measure $\nu$ on $X$. Let $x \in X$ be a point with trivial stabilizer. Then $\delta_{x} \in \operatorname{Prob}(X)$ has a trivial stabilizer. Since $G \curvearrowright X$ is a boundary action, $\delta_{x}$ belongs to the weak*-closure of $G \nu$. Proposition 5.3.ii applied to the action $G \curvearrowright \operatorname{Prob}(X)$ shows that Items (i) and (ii) hold, since $H \subset G_{\nu}$.

Remark 6.7. We will give below (Theorem 7.2) a much stronger version of Theorem 6.6.ii.

A subgroup $H \in \operatorname{Sub}(G)$ is recurrent if $\{e\}$ does not belong to the closure of $\mathcal{C}(H)$ for the Chabauty topology; this notion was introduced in [Ken] and used there (see Theorem 1.1) to give the following characterization of $C^{*}$-simplicity: a discrete group $G$ is $C^{*}$-simple if and only if $G$ has no amenable recurrent subgroup. Since amenable subgroups are weakly parabolic (provided $\operatorname{Sub}_{\mathrm{w}-\mathrm{par}}(G) \neq \emptyset$ ), the following corollary of Theorem 6.6 strengthens one implication in this result.

Corollary 6.8. Let $G$ be a countable group. Then $\operatorname{Sub}_{\mathrm{w}-\mathrm{par}}(G)$ contains no recurrent subgroup of $G$.

\section{7. $C^{*}$-ALGEBRAS ASSOCIATED TO INDUCED REPRESENTATIONS}

We are going to draw some consequences of the results from Section 4 for the primitive ideal space of a countable group $G$; we also study the ideal theory of $C^{*}$-algebras associated to induced representations of 
$G$ from subgroups which either have the spectral gap property or are weakly parabolic.

7.1. Primitive ideal space of $G$. Recall that the primitive ideal space $\operatorname{Prim}(G)$ of $G$ is the set of equivalence classes of irreducible unitary representations of $G$ for the relation of weak equivalence.

Let $C^{*}(G)$ be the maximal $C^{*}$-algebra of $G$ (see Subsection 2.2). One may describe $\operatorname{Prim}(G)$ as the set $\left\{C^{*} \operatorname{ker}(\pi) \mid \pi \in \widehat{G}\right\}$, where $C^{*} \operatorname{ker}(\pi)$ is the kernel of the extension of the representation $\pi$ to $C^{*}(G)$; for all this, see [Dix77, Chap.13].

Let

$$
X:=\left\{(H, \sigma) \mid H \in \operatorname{Sub}_{\mathrm{sc}}(G), \sigma \in \widehat{H}_{f d}\right\},
$$

where $\operatorname{Sub}_{\mathrm{sc}}(G)$ is the set of self-commensurating subgroups of $G$. Recall from Subsection 2.6 that $G$ acts naturally on $X$ and that the map

$$
\text { Ind }: X \rightarrow \operatorname{Rep}(G), \quad(H, \sigma) \mapsto \operatorname{Ind}_{H}^{G} \sigma
$$

factorizes to an injective map $X / G \rightarrow \widehat{G}$, where $X / G$ is the space of orbits for that the natural $G$-action on $X$. This yields the map

$$
X \rightarrow \operatorname{Prim}(G), \quad(H, \sigma) \mapsto C^{*} \operatorname{ker}\left(\operatorname{Ind}_{H}^{G} \sigma\right)
$$

which factorizes through $X / G$.

Items (ii) and (iii) of Theorem 4.3 may be rephrased in terms of the primitive ideal space of $G$ in the following way.

Theorem 7.1. Let $G$ be a countable group. The restriction of the map

$$
X \rightarrow \operatorname{Prim}(G), \quad(H, \sigma) \mapsto C^{*} \operatorname{ker}\left(\operatorname{Ind}_{H}^{G} \sigma\right)
$$

to the G-invariant subset

$$
X_{\mathrm{sg}}:=\left\{(H, \sigma) \mid H \in \operatorname{Sub}_{\mathrm{sg}}(G), \sigma \in \widehat{H}_{\mathrm{fd}}\right\}
$$

factorizes to an injective $\operatorname{map} X_{\mathrm{sg}} / G \rightarrow \operatorname{Prim}(G)$.

Moreover, if $C^{*} \operatorname{ker}(\pi)=C^{*} \operatorname{ker}\left(\operatorname{Ind}_{H}^{G} \sigma\right)$ for some $\pi \in \widehat{G}$ and $(H, \sigma) \in$ $X_{\mathrm{sg}}$, then $\pi$ is equivalent to $\operatorname{Ind}_{H}^{G} \sigma$.

7.2. $C^{*}$-algebras associated to induced representations. We prove a result about the ideal structure of the $C^{*}$-algebra generated by an induced representation from a weakly parabolic subgroup.

In the sequel, by an ideal in a $C^{*}$-algebra $\mathcal{A}$, we mean a closed twosided ideal of $\mathcal{A}$.

Theorem 7.2. Let $G$ be a countable group, $H \in \operatorname{Sub}_{\mathrm{w}-\mathrm{par}}(G)$ and $\sigma \in \operatorname{Rep}(H)$. Let $\pi:=\operatorname{Ind}_{H}^{G} \sigma$. The $C^{*}$-algebra $C_{\pi}^{*}(G)$ contains a 
(unique) largest proper ideal $I_{\max }$ that is, a proper ideal of $C_{\pi}^{*}(G)$ which contains every proper ideal of $C_{\pi}^{*}(G)$. Moreover, the map

$$
\pi(G) \rightarrow \lambda_{G}(G), \quad \pi(g) \mapsto \lambda_{G}(g)
$$

extends to a surjective $*$-homomorphism $C_{\pi}^{*}(G) \rightarrow C_{\mathrm{red}}^{*}(G)$ with kernel $I_{\max }$.

Proof. Let $\mathcal{K}$ be the Hilbert space of $\sigma$. Recall that the Hilbert space $\mathcal{H}$ of $\pi=\operatorname{Ind}_{H}^{G} \sigma$ is the space of maps $F: G \rightarrow \mathcal{K}$ such that $F(x h)=$ $\sigma\left(h^{-1}\right) F(x)$ for all $x \in G, h \in H$ and such that $x H \mapsto\|F(x)\|$ belongs to $\ell^{2}(G / H)$. Every $f \in \ell^{\infty}(G / H)$ defines a bounded operator $M(f)$ on $\mathcal{H}$, given by multiplication by $f$ :

$$
(M(f) F)(x)=f(x H) F(x) \quad \text { for all } \quad x \in G, F \in \mathcal{H} ;
$$

moreover, we have the covariance relation

$$
\pi(g) M(f) \pi\left(g^{-1}\right)=M\left({ }_{g} f\right) \quad \text { for all } \quad g \in G,
$$

where ${ }_{g} f \in \ell^{\infty}(G / H)$ is defined by ${ }_{g} f(x H)=f\left(g^{-1} x H\right)$.

Since $H \in \operatorname{Sub}_{\mathrm{w}-\mathrm{par}}(G)$, there exist a topologically free boundary action $G \curvearrowright X$ and a linear isometric map

$$
P: C(X) \rightarrow \ell^{\infty}(G / H)
$$

which is $G$-equivariant, unital and positive (see Proposition 6.4). Observe that $P$ is not an algebra homomorphism in general. We will view $C(X)$ as a closed $G$-invariant subspace of $\ell^{\infty}(G / H)$.

Let $S\left(C_{\pi}^{*}(G)\right)$ and $S(\mathcal{B}(\mathcal{H}))$ be the state spaces of $C_{\pi}^{*}(G)$ and $\mathcal{B}(\mathcal{H})$ (see Subsection 2.4). The action $G \curvearrowright S\left(C_{\pi}^{*}(G)\right)$ extends to an action $G \curvearrowright S(\mathcal{B}(\mathcal{H}))$, given by the same formula:

$$
g \cdot \varphi(T)=\varphi\left(\pi(g) T \pi\left(g^{-1}\right)\right) \quad \text { for } \quad g \in G, \varphi \in S(\mathcal{B}(\mathcal{H})), T \in \mathcal{B}(\mathcal{H}) .
$$

Recall that the embedding $P: C(X) \rightarrow \ell^{\infty}(G / H)$ preserves positivity. Hence, for $\varphi \in S(\mathcal{B}(\mathcal{H}))$, the map

$$
C(X) \rightarrow \mathbf{C}, \quad f \mapsto \varphi(M(f)),
$$

denoted by $\left.\varphi\right|_{C(X)}$, is a state on $C(X)$ and is therefore given by a probability measure on $X$. So, $\left\{\left.\varphi\right|_{C(X)} \mid \varphi \in S(\mathcal{B}(\mathcal{H}))\right\}$ can be identified with $\operatorname{Prob}(X)$; moreover, since $C(X)$ is $G$-equivariantly embedded in $\ell^{\infty}(G / H)$ and in view of the relation $(*)$, the restriction of the action $G \curvearrowright S(\mathcal{B}(\mathcal{H}))$ to $\operatorname{Prob}(X)$ under this identification corresponds to the natural action of $G$ on $\operatorname{Prob}(X)$.

From now on, we imitate some of the arguments given in the proof of [Haa15, Theorem 4.5], adapting them to our context. 
- Step 1. Let $\varphi \in S\left(C_{\pi}^{*}(G)\right)$ and $x \in X$. Let $\varphi^{\prime} \in S(\mathcal{A})$ be an extension of $\varphi$. There exists a net $\left(g_{i}\right)_{i}$ in $G$ such that $\left(g_{i} \cdot \varphi^{\prime}\right)_{i}$ converges to a state $\psi \in S(\mathcal{A})$ with $\left.\psi\right|_{C(X)}=\delta_{x}$.

Indeed, let $\mu=\left.\varphi^{\prime}\right|_{C(X)} \in \operatorname{Prob}(X)$. Since $G \curvearrowright X$ is a boundary action, there exists a net $\left(g_{i}\right)_{i}$ in $G$ such that $\lim _{i} g_{i} \cdot \mu=\delta_{x}$. By compactness of $S(\mathcal{A})$, by passing to a subnet if necessary, we can assume that $\left(g_{i} \cdot \varphi^{\prime}\right)_{i}$ converges to some $\psi \in S(\mathcal{A})$. Then $\left.\lim _{i}\left(g_{i} \cdot \varphi^{\prime}\right)\right|_{C(X)}=\left.\psi\right|_{C(X)}$ and, since $\left.\left(g_{i} \cdot \varphi^{\prime}\right)\right|_{C(X)}=g_{i} \cdot \mu$, we have $\left.\psi\right|_{C(X)}=\delta_{x}$.

- Step 2. Let $\varphi \in S\left(C_{\pi}^{*}(G)\right)$ and $x \in X$. Assume that $g x \neq x$ for every $g \in G \backslash\{e\}$. There exists a state $\psi \in S\left(C_{\pi}^{*}(G)\right)$ which belongs to the weak* closure of $\{g \cdot \varphi \mid g \in G\}$ such that

$$
\psi(\pi(g))=0 \quad \text { for all } g \in G \backslash\{e\} .
$$

Indeed, let $\varphi^{\prime} \in S(\mathcal{A}), g_{i} \in G$ and $\psi \in S(\mathcal{A})$ be as in Step 1. Let $g \in G \backslash\{e\}$. Since $g x \neq x$, we can choose $f \in C(X)$ such that

$$
0 \leq f \leq \mathbb{1}_{C(X)}, \quad f(g x)=0, \quad \text { and } \quad f(x)=1 .
$$

Hence, we have $0 \leq 1-f \leq \mathbb{1}_{C(X)}$ and so

$$
0 \leq M\left(\mathbb{1}_{C(X)}-f\right) \leq M\left(\mathbb{1}_{C(X)}\right) .
$$

Therefore,

$$
\left(M\left(\mathbb{1}_{C(X)}-f\right)\right)^{2} \leq M\left(\mathbb{1}_{C(X)}-f\right) .
$$

One the one hand, using the Cauchy-Schwartz inequality, it follows that

$$
\begin{aligned}
\left|\psi\left(\left(\mathbb{1}_{\mathcal{B}(\mathcal{H})}-M(f)\right) \pi(g)\right)\right|^{2} & =\left|\psi\left(\left(M\left(\mathbb{1}_{C(X)}-f\right)\right) \pi(g)\right)\right|^{2} \\
& \leq \psi\left(\left(M\left(\mathbb{1}_{C(X)}-f\right)\right)^{2}\right) \psi\left(\mathbb{1}_{\mathcal{B}(\mathcal{H})}\right) \\
& \leq \psi\left(M\left(\mathbb{1}_{C(X)}-f\right)\right) \\
& =1-\psi(M(f)) \\
& =1-f(x)=0,
\end{aligned}
$$

so that $\psi(\pi(g))=\psi(M(f) \pi(g))$.

On the other hand, since $0 \leq f \leq \mathbb{1}_{C(X)}$, we have, as above,

$$
M(f)^{2} \leq M(f) .
$$

Using the fact that $\psi\left(T^{*}\right)=\overline{\psi(T)}$ for any $T \in \mathcal{B}(\mathcal{H})$ and applying again the Cauchy-Schwartz inequality, it follows that

$$
\begin{aligned}
|\psi(M(f) \pi(g))|^{2} & \left.=\mid \psi\left(\pi\left(g^{-1}\right) M(f)\right)\right)\left.\right|^{2} \\
& \leq \psi\left(\pi\left(g^{-1}\right) M(f)^{2} \pi(g)\right) \\
& \leq \psi\left(\pi\left(g^{-1}\right) M(f) \pi(g)\right)
\end{aligned}
$$




$$
\begin{aligned}
& =\psi\left(M\left(g^{-1} f\right)\right) \\
& =f(g x)=0 ;
\end{aligned}
$$

so, $\psi(M(f) \pi(g))=0$. Hence, $\psi(\pi(g))=0$, as claimed.

Recall (see Subsection 2.4) that we can view $S\left(C_{\rho}^{*}(G)\right)$ as a weak* closed subset of $S\left(C^{*}(G)\right)$ for every $\rho \in \operatorname{Rep}(G)$.

- Step 3. Let $\varphi \in S\left(C_{\pi}^{*}(G)\right)$. The canonical trace $\tau_{G}$ belongs to the weak*-closure of $\{g \cdot \varphi \mid g \in G\}$.

Indeed, since $G \curvearrowright X$ is topologically free, we can find $x \in X$ with $g x \neq x$ for every $g \in G \backslash\{e\}$. Let $\psi \in S\left(C_{\pi}^{*}(G)\right)$ be as in Step 2. Then $\psi(\pi(g))=0$ for every $g \in G \backslash\{e\}$. Hence, $\psi=\tau_{G}$, when $\psi$ and $\tau_{G}$ are viewed as elements of $S\left(C^{*}(G)\right)$. This proves the claim.

- Step 4. Let $\rho \in \operatorname{Rep}(G)$ be such that $\rho \prec \pi$. Then $\lambda_{G} \prec \rho$.

Indeed, let $\varphi \in S\left(C_{\rho}^{*}(G)\right)$. Since $\rho \prec \pi$, we can view $\varphi$ as a state on $C_{\pi}^{*}(G)$. Hence, $\tau_{G}$ belongs to the weak*-closure of $\{g \cdot \varphi \mid g \in G\}$, by Step 3. As $g \cdot \varphi \in S\left(C_{\rho}^{*}(G)\right)$, it follows that $\tau_{G} \in S\left(C_{\rho}^{*}(G)\right)$; hence, $\lambda_{G} \prec \rho$, since $\lambda_{G}$ is cyclic.

In particular, it follows from Step 4 that $\lambda_{G} \prec \pi$ and so the map $\pi(g) \mapsto \lambda_{G}(g)$, extends to a surjective $*$-homomorphism $C_{\pi}^{*}(G) \rightarrow$ $C_{\text {red }}^{*}(G)$. Let $I$ be the kernel of this $*$-homomorphism. So, $C_{\pi}^{*}(G) / I$ is isomorphic to $C_{\text {red }}^{*}(G)$.

- Step 5. We claim that $I$ is a proper ideal of $C_{\pi}^{*}(G)$ that contains all other proper ideals of $C_{\pi}^{*}(G)$.

Indeed, observe first that $I$ is proper since it is the kernel of a nonzero homomorphism. Now, let $J$ be a proper ideal of $C_{\pi}^{*}(G)$. Then $J$ is the kernel of the extension to $C_{\pi}^{*}(G)$ of a unitary representation $\rho$ of $G$. Then $\rho \prec \pi$ and hence, by Step $4, \lambda_{G} \prec \rho$. Therefore, $J \subset I$.

Remark 7.3. (i) Let $G$ be a countable group, $H \in \operatorname{Sub}_{\mathrm{w}-\mathrm{par}}(G)$, and $\sigma \in \operatorname{Rep}(H)$. Then, for the ideal $I_{\max }$ as in Theorem 7.2, we have

$$
I_{\text {max }}=\{0\} \Longleftrightarrow \sigma \prec \lambda_{H} .
$$

Indeed, since $\lambda_{G} \prec \operatorname{Ind}_{H}^{G} \sigma$, we have (see Corollary 2.3)

$$
I_{\max }=\{0\} \Longleftrightarrow \operatorname{Ind}_{H}^{G} \sigma \prec \lambda_{G} \Longleftrightarrow \sigma \prec \lambda_{H} .
$$

In particular, for $\sigma=1_{H}$ so that $\operatorname{Ind}_{H}^{G} \sigma=\lambda_{G / H}$, we have $I_{\max }=\{0\}$ if and only if $H$ is amenable.

(ii) Let $G$ be a countable group such that there exists a topologically free boundary action $G \curvearrowright X$. Then Theorem 7.2 applied to the case $H=\{e\} \in \operatorname{Sub}_{\mathrm{w}-\text { par }}(G)$ shows that that $C_{\text {red }}^{*}(G)$ is simple. Thus, Theorem 7.2 generalizes the main criterium for $C^{*}$-simplicity of $G$ from [KK17] (see Theorem 5.6 above). 
(iii) Let $G$ be a countable group, $H \in \operatorname{Sub}_{\mathrm{w}-\operatorname{par}}(G)$ and $\pi=\operatorname{Ind}_{H}^{G}$ for $\sigma \in \operatorname{Rep}(H)$. Using arguments as in the proof of [Haa15, Theorem 4.5], one can rephrase Theorem 7.2 in terms of a "Dixmier approximation property": for every finite subset $F$ of $G \backslash\{e\}$ and $\varepsilon>0$, there exist elements $g_{1}, \ldots, g_{n} \in G$ such that

$$
\left\|\frac{1}{n} \sum_{i=1}^{n} \pi\left(g_{i} g g_{i}^{-1}\right)\right\|<\varepsilon \quad \text { for every } g \in F \text {. }
$$

Next, we examine a consequence of Theorem 4.3 for the ideal theory of the $C^{*}$-algebra generated by an induced representation from a subgroup with the spectral gap property.

Theorem 7.4. Let $G$ be a countable group, $H \in \operatorname{Sub}_{\mathrm{sg}}(G)$ and $\sigma \in$ $\widehat{H}_{\text {fin. }}$. Let $\pi:=\operatorname{Ind}_{H}^{G} \sigma$. The $C^{*}$-algebra $C_{\pi}^{*}(G)$ contains a (unique) smallest non-zero ideal $I_{\min }$, that is, a non-zero ideal such that every non-zero ideal $C_{\pi}^{*}(G)$ contains $I_{\min }$.

Proof. Theorem 4.3 shows that $\pi$ is a traceable irreducible representation; hence, $C_{\pi}^{*}(G)$ contains the ideal $I=\mathcal{K}(\mathcal{H})$ of compact operators on the Hilbert space $\mathcal{H}$ of $\pi$. Let $J$ be a non-zero ideal of $C_{\pi}^{*}(G)$. Then clearly $J I \neq\{0\}$ and hence $J \cap I \neq\{0\}$. Since $I$ is a simple $C^{*}$-algebra, it follows that $I \subset J$.

For subgroups $H$ belonging to both $\operatorname{Sub}_{\mathrm{sg}}(G)$ and $\operatorname{Sub}_{\mathrm{w}-\mathrm{par}}(G)$, we have the following consequence of Theorem 7.2 and Theorem 7.4.

Corollary 7.5. Let $G$ be a countable group, $H \in \operatorname{Sub}_{\mathrm{w}-\operatorname{par}}(G) \cap \operatorname{Sub}_{\mathrm{sg}}(G)$, and $\sigma \in \widehat{H}_{\text {fin }}$. Let $\pi:=\operatorname{Ind}_{H}^{G} \sigma$.

(i) The $C^{*}$-algebra $C_{\pi}^{*}(G)$ contains a smallest non-zero ideal.

(ii) The regular representation $\lambda_{G}$ extends to a representation of $C_{\pi}^{*}(G)$ and the kernel of this extension is the largest proper ideal of $C_{\pi}^{*}(G)$.

(iii) There exists $\pi_{\min } \in \operatorname{Rep}(G)$ with the following property: for every $\rho \in \operatorname{Rep}(G)$ with $\rho \prec \pi$ and $\rho \neq \pi$, we have

$$
\lambda_{G} \prec \rho \prec \pi_{\min } \prec \pi .
$$

Proof. Items (i) and (ii) are immediate consequences of Theorem 7.2 and Theorem 7.4 .

Let $\pi_{\min }$ be a representation of $C_{\pi}^{*}(G)$ with kernel the smallest nonzero ideal $I_{\min }$; the restriction of $\pi_{\min }$ to $G$ is a unitary representation, again denoted by $\pi_{\text {min }}$.

Let $\rho \in \operatorname{Rep}(G)$ with $\rho \prec \pi$ and $\rho \neq \pi$. The kernel $J$ of the extension of $\rho$ to $C_{\pi}^{*}(G)$ is a non-zero proper ideal of $C_{\pi}^{*}(G)$. Hence, 
$I_{\min } \subset J \subset I_{\max }$, by Items (i) and (ii), with $I_{\max }$ the kernel of the extension of $\lambda_{G}$ to $C_{\pi}^{*}(G)$; this means that $\lambda_{G} \prec \rho \prec \pi_{\min }$.

Remark 7.6. It is conceivable - but we know of no example - that, for some groups $G$ and subgroups $H \in \operatorname{Sub}_{\text {w-par }}(G) \cap \operatorname{Sub}_{\mathrm{sg}}(G)$, the ideals $I_{\min }$ and $I_{\max }$ of $C_{\lambda_{G / H}}^{*}(G)$ coincide. If this happens, then $C_{\lambda_{G / H}}^{*}(G)$ has a unique non-trivial ideal.

Example 7.7. Let $G=P S L_{n}(\mathbf{Z})$ for $n \geq 3$. Let $H$ be a standard copy of $P S L_{n-1}(\mathbf{Z})$ inside $G$, that is, the image in $G$ of, say,

$$
\left[\begin{array}{cc}
1 & 0 \\
0 & S L_{n-1}(\mathbf{Z})
\end{array}\right]
$$

We claim that $H \in \operatorname{Sub}_{\mathrm{w}-\operatorname{par}}(G) \cap \operatorname{Sub}_{\mathrm{sg}}(G)$.

Indeed, the fact that $H \in \operatorname{Sub}_{\mathrm{w}-\mathrm{par}}(G)$ is shown in Example 6.3, To show that $H \in \operatorname{Sub}_{\mathrm{sg}}(G)$, we have to consider the two cases: $n=3$ and $n \geq 4$.

- $n=3$ : Example 5.17 shows that $H$ is a-normal. Since $H$ is non-amenable, we have $H \in \operatorname{Sub}_{\mathrm{sg}}(G)$, by Proposition 4.11.

- $n \geq 4$ : the subgroup $H$ has Kazhdan's propery $(\mathrm{T})$ since $n-1 \geq$ 3. Moreover, $[1: 0: \cdots: 0]$ is the unique periodic point of $H$ in $X=\mathbf{P}\left(\mathbf{R}^{n}\right)$. It follows that $H$ is strongly self-commensurating. Hence, $H \in \operatorname{Sub}_{\mathrm{sg}}(G)$, by Proposition 4.6.

With similar arguments, it can be shown that every standard copy of $P G L_{n-1}(\mathbf{Q})$ inside $G=P G L_{n}(\mathbf{Q})$ belongs to $\operatorname{Sub}_{\mathrm{w}-\mathrm{par}}(G) \cap \operatorname{Sub}_{\mathrm{sg}}(G)$ for $n \geq 3$.

\section{REFERENCES}

[BB18] B. Bekka, Infinite characters on $G L_{n}(\mathbf{Q})$, on $S L_{n}(\mathbf{Z})$, and on groups acting on trees (2018), available at arXiv:1806.10110.

[BB17] Spectral rigidity of group actions on homogeneous spaces (2017), available at arXiv1602.02892.

[BCdlH94] B. Bekka, M. Cowling, and P. de la Harpe, Simplicity of the reduced $C^{*}$-algebra of $\operatorname{PSL}(n, \mathbf{Z})$, Internat. Math. Res. Notices 7 (1994), 285-291.

[BdlHV08] B. Bekka, P. de la Harpe, and A. Valette, Kazhdan's property (T), New Mathematical Monographs, vol. 11, Cambridge University Press, Cambridge, 2008.

[Bér92] P. Bérard, Transplantation et isospectralité. I, Math. Ann. 292 (1992), no. 3, 547-559.

[BPR14] C. Bhagwat, S. Pisolkar, and C. S. Rajan, Commensurability and representation equivalent arithmetic lattices, Int. Math. Res. Not. IMRN 8 (2014), 2017-2036.

[Bow98] B. H. Bowditch, A topological characterisation of hyperbolic groups, J. Amer. Math. Soc. 11 (1998), no. 3, 643-667. 
[Bow12] _ Relatively hyperbolic groups, Internat. J. Algebra Comput. 22 (2012), no. 3, 1250016, 66.

[BKKO17] E. Breuillard, M. Kalantar, M. Kennedy, and N. Ozawa, $C^{*}$-simplicity and the unique trace property for discrete groups, Publ. Math. Inst. Hautes Études Sci. 126 (2017), 35-71.

[CFP96] J. W. Cannon, W. J. Floyd, and W. R. Parry, Introductory notes on Richard Thompson's groups, Enseign. Math. (2) 42 (1996), no. 3-4, 215-256.

[CM14] P.-E Caprace and N. Monod, Relative amenability, Groups Geom. Dyn. 8 (2014), no. 3, 747-774.

[Cha50] C. Chabauty, Limite d'ensembles et géométrie des nombres, Bull. Soc. Math. France 78 (1950), 143-151.

[Cor75] L. Corwin, Induced representations of discrete groups, Proc. Amer. Math. Soc. 47 (1975), 279-287.

[Dix77] J. Dixmier, $C^{*}$-algebras, North-Holland Publishing Co., Amsterdam-New York-Oxford, 1977.

[Eff63] Edward G. Effros, A decomposition theory for representations of $C^{*}$ algebras, Trans. Amer. Math.Soc. 107 (1963), 83-106.

[Fel64] J. M. G. Fell, Weak containment and induced representations of groups. II, Trans. Amer. Math. Soc. 110 (1964), 424-447.

[Fro71] Z. Frolík, Maps of extremally disconnected spaces, theory of types, and applications, General Topology and its Relations to Modern Analysis and Algebra (1971), 131-142.

[Fur73] H. Furstenberg, Boundary theory and stochastic processes on homogeneous spaces, Harmonic analysis on homogeneous spaces, Proceedings of the Symposium of Pure Mathematics, vol. 26, American Mathematical Society, Providence, RI., 1973.

[Gla15] E. and Weiss Glasner B., Uniformly recurrent subgroups, Recent trends in ergodic theory and dynamical systems, 2015, pp. 63-75.

[Gli61] J. Glimm, Type I $C^{*}$-algebras, Ann. of Math. (2) 73 (1961), 572-612.

[Gor09] C. Gordon, Sunada's isospectrality technique: two decades later, Spectral analysis in geometry and number theory, Contemp. Math., vol. 484, Amer. Math. Soc., Providence, RI, 2009, pp. 45-58.

[Haa15] U. Haagerup, A new look at $C^{*}$-simplicity and the unique trace property of a group (2015), available at arXiv: 1509.05880.

[KK17] M. Kalantar and M. Kennedy, Boundaries of reduced $C^{*}$-algebras of discrete groups, J. Reine Angew. Math. (Crelle's Journal) 727 (2017), 247-267.

[KB02] I. Kapovich and N. Benakli, Boundaries of hyperbolic groups, Combinatorial and geometric group theory (New York, 2000/Hoboken, NJ, 2001), Contemp. Math., vol. 296, Amer. Math. Soc., Providence, RI, 2002, pp. 39-93, DOI 10.1090/conm/296/05068. MR1921706

[Ken] M. Kennedy, An intrinsic characterization of $C^{*}$-simplicity, Ann. Sci. École Norm. Sup., to appear.

[Kle61] A. Kleppner, On the intertwining number theorem, Proc. Amer. Math. Soc. 12 (1961), 731-733.

[LB17] A. Le Boudec, $C^{*}$-simplicity and the amenable radical, Invent. Math. 209 (2017), no. 1, 159-174. 
[LBMB18] A. Le Boudec and N. Matte Bon, Subgroup dynamics and $C^{*}$-simplicity of groups of homeomorphisms, Ann. Sci. Éc. Norm. Supér. (4) 51 (2018), no. 3, 557-602.

[LS01] R. C. Lyndon and P. E. Schupp, Combinatorial group theory, Classics in Mathematics, Springer-Verlag, Berlin, 2001. Reprint of the 1977 edition.

[Mac51] G. W. Mackey, On induced representations of groups, Amer. J. Math. 73 (1951), 576-592.

[PS79] W. L. Paschke and N. Salinas, $C^{*}$-algebras associated with free products of groups, Pacific J. Math. 82 (1979), no. 1, 211-221.

[PV08] S. Popa and S. Vaes, Strong rigidity of generalized Bernoulli actions and computations of their symmetry groups, Adv. Math. 217 (2008), no. 2, 833872.

[Sun85] T. Sunada, Riemannian coverings and isospectral manifolds, Ann. of Math. (2) 121 (1985), no. 1, 169-186.

[Tho68] E. Thoma, Eine Charakterisierung diskreter Gruppen vom Typ I, Invent. Math. 6 (1968), 190-196.

[Wil07] D. P. Williams, Crossed products of $C^{*}$-algebras, Mathematical Surveys and Monographs, vol. 134, American Mathematical Society, Providence, RI, 2007.

[Yam04] A. Yaman, A topological characterisation of relatively hyperbolic groups, J. Reine Angew. Math. 566 (2004), 41-89.

Bachir Bekka, Univ Rennes, CNRS, IRMaR-UMR 6625, Campus Beaulieu, F-35042 Rennes Cedex, France

E-mail address: bachir.bekka@univ-rennes1.fr

Mehrdad Kalantar, Department of Mathematics, University of HousTON, Houston, TX, USA

E-mail address: kalantar@math.uh.edu 